\title{
Phosphorus-nitrogen compounds. Part 41. Ferrocenyl pendant-armed spirocyclopiperidinocyclotriphosphazatrienes: Langmuir-Blodgett thin films and biological activity studies
}

\author{
NURAN ASMAFILIZZ ${ }^{\mathrm{a}, *}$ (D) MEHMET CIVAN ${ }^{\mathrm{b}}$, NEŞE UZUNALIOĞLUU $^{\mathrm{a}}$, ARDA ÖZBEN $^{\mathrm{a}}$, \\ ZEYNEL KILIÇa ${ }^{\mathrm{a}}$, HANDE KAYALAK ${ }^{\mathrm{c}}$, LEYLA AÇIK ${ }^{\mathrm{c}}$ and TUNCER HÖKELEK ${ }^{\mathrm{b}}$ \\ ${ }^{a}$ Department of Chemistry, Ankara University, 06100 Ankara, Turkey \\ ${ }^{b}$ Department of Physics, Hacettepe University, 06800 Ankara, Turkey \\ ${ }^{c}$ Department of Biology, Gazi University, 06500 Ankara, Turkey \\ E-mail: gurun@science.ankara.edu.tr
}

MS received 20 April 2018; revised 25 July 2018; accepted 27 July 2018; published online 30 October 2018

\begin{abstract}
The $\mathrm{Cl}$ replacement reactions of N/N or N/O spirocyclic monoferrocenylcyclotriphosphazatrienes (1-5) with the piperidine resulted in the geminal- (6-10) and tetra-piperidinophosphazenes with monoferrocenyl pendant arm (11-15). The structures of all the new compounds were determined using spectroscopic techniques. The ultrathin Langmuir-Blodgett (LB) films of two compounds ( $\mathbf{3}$ and 12) were prepared. The characterization of the LB films using p-polarized grazing angle (GAIR) and horizontal attenuated total reflectance (HATR) techniques was carried out. The molecular and crystal structure of the compound $\mathbf{6}$ was examined using X-ray crystallography. In addition, the interactions between six compounds $(\mathbf{6}, \mathbf{7}, \mathbf{1 1}, \mathbf{1 2}, 14$ and 15) and pBR322 plasmid DNA were investigated by agarose gel electrophoresis.
\end{abstract}

Keywords. Pendant-armed ferrocenyl phosphazenes; spectroscopy; thin films; DNA cleavage.

\section{Introduction}

The chemistry of hexachlorocyclotriphosphazenes $\left(\mathrm{N}_{3}\right.$ $\mathrm{P}_{3} \mathrm{Cl}_{6}$; trimer) and octachlorocyclotetraphosphazenes $\left(\mathrm{N}_{4} \mathrm{P}_{4} \mathrm{Cl}_{8}\right.$; tetramer $)$ has been widely studied since 1960. ${ }^{1}$ Trimer and tetramer are used as scaffolds for the construction of numerous substituted cyclotriphosphazenes. ${ }^{2}$ The sequential $\mathrm{Cl}$ replacement reactions of trimer and tetramer with mono-functional reagents produce the partly and fully-substituted phosphazenes. ${ }^{3}$ However, the reactions carried out with di-, tri- and multi-functional reagents give different geometrical and optical isomers, e.g., spiro-, ansa-, dispiro-, trispiro, ansa-spiro and bino-architectures. ${ }^{2-4}$ In addition, trimer and tetramer are also used as building blocks for dendrimers ${ }^{5}$ and phosphazene polymers. ${ }^{6}$ In recent years, the coordination complexes of the aminocyclophosphazene have attracted interest as an active research area. ${ }^{7}$ In the last two decades, the stereogenic properties of cyclophosphazenes have been widely investigated, ${ }^{8}$ and recently a nice review was published as well. ${ }^{9}$ Although the stereochemical terms such as prochirality, enantio-, diastereo- and homotopic atoms or substituents and pseudo-asymmetric centers were known for a long time in organic chemistry, they were incorporated into phosphazene derivatives only in two papers. ${ }^{10}$ On the other hand, phosphazene derivatives and polymeric phosphazenes are used as inflammable textile fibers and elastomers, ${ }^{11}$ antibacterial ${ }^{12}$ and anticancer ${ }^{13}$ reagents, lubricants, ${ }^{14}$ membranes, ${ }^{15}$ synthetic bones, ${ }^{16}$ ion-transferring agents for rechargeable lithium batteries ${ }^{17}$ and photophysic. ${ }^{18}$ Ferrocene $(\mathrm{Fc})$ derivatives are used in organometallic chemistry, ${ }^{19}$ photochemistry, ${ }^{20}$ redox-active probe materials ${ }^{21}$ and electron transfer mediators for nonlinear optical devices ${ }^{22}$ as well.

In the meantime, the ultrathin films of inorganic and organic materials were obtained with different methods such as thermal evaporation, adsorption from solution, electrodeposition, sputtering, self-assembly, molecular beam epitaxy and Langmuir-Blodgett (LB)

\footnotetext{
*For correspondence

Electronic supplementary material: The online version of this article (https://doi.org/10.1007/s12039-018-1545-x) contains supplementary material, which is available to authorized users.
} 
techniques. ${ }^{23}$ The LB films are very useful materials in industry e.g., as detectors, sensors and electronic circuit components. ${ }^{24}$ Our group published two papers about the LB thin films of trimeric phosphazene derivatives. ${ }^{25}$

The literature survey shows that there are very limited number of reports about the pendant armed spirocyclotriphosphazenes, their reactions, and spectral and structural properties. ${ }^{26}$ Actually, several papers have been reported by our group. ${ }^{3,4 a, 27}$

This paper deals with the condensation reactions of the tetrachloro monoferrocenyl pendant-armed spirophosphazenes (1-5) with piperidine for determining their biological activities and DNA interactions, and also for preparing the LB thin films of the partly and fullypiperidino-substituted compounds. Thus, the bis- (6-10) and tetrakis-piperidino-substituted (11-15) N/N and N/O spirocyclotriphosphazenes with monoferrocenyl pendant arm were obtained from the substitution reactions. The structures of all the new phosphazenes $(\mathbf{6}-\mathbf{1 5})$ were analyzed using mass spectrometry, elemental analyses, Fourier transform (FTIR) and one-dimensional (1D) ${ }^{1} \mathrm{H},{ }^{13} \mathrm{C}\left\{{ }^{1} \mathrm{H}\right\}$, and ${ }^{31} \mathrm{P}\left\{{ }^{1} \mathrm{H}\right\}$ NMR techniques. The molecular and crystal structures of $\mathbf{6}$ were established by X-ray crystallography. The purpose of combining the trimer with the ferrocenyl group is to create compounds with new properties from these two groups. According to our previous studies, ${ }^{3 \mathrm{a}, 8 \mathrm{c}, 25 \mathrm{~b}}$ it has been observed that geminal products have occurred from the reactions of the ferrocenylphosphazenes with the secondary amines. So, ferrocenyl pendant-armed spiro precursors have also been chosen for the formation of geminal products instead of non-geminal products with piperidine. In addition, piperidine substituents increase the basic character of the phosphazene ring as well. As understood in this study, these novel compounds may be used for different purposes in the future in addition to the above-mentioned uses, taking into account their different properties. To confirm this view, due to the difficulties in preparing LB films of all compounds, only two phosphazenes $\mathbf{3}$ and $\mathbf{1 2}$ were selected as examples and LB films were prepared. The LB films of other analog phosphazenes can also be prepared.

\section{Experimental}

\subsection{Reagents}

Ferrocenecarboxaldehyde (Aldrich), hexachlorocyclotriphosphazatriene (Aldrich) and aliphatic amines (Fluka) were purchased and used without further purification. THF was dried over $3 \AA$ molecular sieves. All the reactions were monitored with thin-layer chromatography (TLC) on Merck DC Alufolien Kiesegel $60 \mathrm{~B}_{254}$ sheets using toluene as solvent. The column chromatography was performed on Merck Kiesegel 60 (230-400 mesh ATSM) silica gel purchased and used without further purification.

\subsection{Instruments}

The melting points were measured with a Gallenkamp apparatus using a capillary tube. The elemental analyses were performed by Leco CHNS-932. The ${ }^{1} \mathrm{H},{ }^{13} \mathrm{C}$ and ${ }^{31} \mathrm{P}$ NMR spectra were recorded on a Varian Mercury FT-NMR spectrometer $\left(\mathrm{SiMe}_{4}\right.$ as an internal standard and $85 \% \mathrm{H}_{3} \mathrm{PO}_{4}$ as an external standard), operating at 400.00, 100.59 and $161.92 \mathrm{MHz}$. The spectrometer was equipped with a $5 \mathrm{~mm}$ PABBO BB inverse-gradient probe. Standard Bruker pulse programs were used. ${ }^{28}$ APIES mass spectrometric analyses were performed on a Waters 2695 Alliance Micromass ZQ spectrometer. IR spectra with $\mathrm{KBr}$ pellet were recorded on a Perkin-Elmer Fourier Transform Infrared (FTIR) Spectrometer equipped with transmission accessories. A commercially available computer-controlled round alternate trough manufactured by NIMA (Model TKB 2410A) was used for the preparation of the LB films. In order to elucidate the structures of the LB films, FTIR, p-polarized GAIR and HATR spectra were recorded. Transmittance spectra at normal incidence and p-polarized GAIR spectra at an incidence angle of $82^{\circ}$ were measured on a Perkin Elmer Spectrum One FTIR Spectrometer equipped with a Deuterated Triglycine Sulfate (DTGS) detector. A ZnSe polarizer was employed for the polarization measurements of the LB films. Both GAIR and HATR spectra were collected for 1000 interferograms with a resolution of $4 \mathrm{~cm}^{-1}$ using the same sample. A Perkin Elmer horizontal attenuated total reflectance attachment was used for HATR measurements. The angle of incidence was $45^{\circ}$ with $\mathrm{ZnSe}$ crystal. In this technique, LB film on aluminum covered glass substrate was pressed on $\mathrm{ZnSe}$ crystal using a very sensitive computer controlled pressure arm to obtain a good contact between the sample and the infrared element (ZnSe crystal) at the interface that was essential requisite for the technique. ${ }^{29}$

Antimicrobial susceptibility testing was performed by the BACTEC MGIT 960 (Becton Dickinson, Sparks, MD) system using the agar-well diffusion method (Section S1, Supplementary Information). The DNA binding abilities were examined using agarose gel electrophoresis (Section S2, Supplementary Information).

\subsection{LB film deposition}

The floating Langmuir monolayers of $\mathbf{3}$ and $\mathbf{1 2}$ were obtained in pure chloroform $(3.5$ and $3.6 \mathrm{mg} / \mathrm{mL})$. The investigations of the surface pressure-area $(\pi-\mathrm{A})$ isotherms, the Langmuir monolayers at the air-water interface and the stabilities of the monolayer of $\mathbf{3}$ and $\mathbf{1 2}$ were performed. To determine these parameters, the solutions of the compounds in chloroform were added dropwise $(62 \mu \mathrm{L})$ using a Hamilton microsyringe on to the subphase surface. After that, for the evaporation of the solvent, the solution waited $2-3 \mathrm{~min}$. The $\pi-\mathrm{A}$ isotherms were obtained with a compression rate of $50 \mathrm{~cm}^{2} \mathrm{~min}^{-1}$ until 


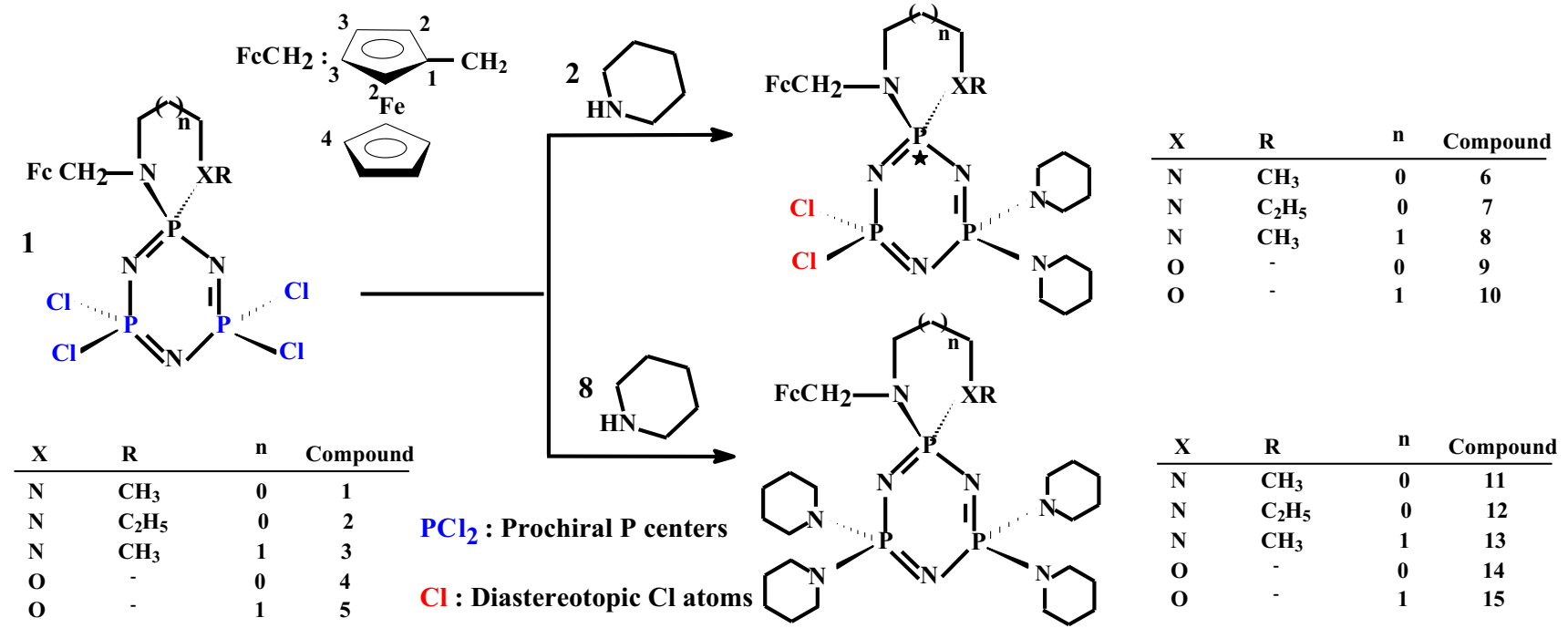

Scheme 1. The $\mathrm{Cl}$ replacement reaction pathway of monoferrocenyl-cyclotriphosphazenes.

the given surface pressure value was reached. The Wilhelmy Method was used for the measure. The estimated standard deviations were found to be $\pm 0.1 \mathrm{mN} \mathrm{m}^{-1}$ for pressure and $\pm 0.1 \mathrm{~cm}^{2}$ for the area. While the targeted pressure was reached, after approximately $17 \mathrm{~min}$, the stable monolayer occurred. The monolayer was stable for $1.5 \mathrm{~h}$. This stability or the surface equilibrium at the interface is very important because of the ordered film deposition. Otherwise, a little change of this parameter affects the whole deposition process. The LB films of $\mathbf{3}$ and $\mathbf{1 2}$ on the substrates were obtained with the thicknesses of four monolayers. The substrates were glass slide coated with a $50 \mathrm{~nm}$ film of thermally evaporated aluminium. The dipping rate was adjusted to $10 \mathrm{~mm} \mathrm{~min}^{-1}$ when the deposition was performed. The LB films were deposited by only upstroke at ambient temperature. Thus, all the transferred monolayers were Z-type.

\section{$2.4 \quad X$-ray crystallography}

Suitable crystals of compounds (3, 6 and 12) were obtained from acetonitrile at room temperature. Crystallographic data were recorded on a Bruker Kappa APEXII CCD area-detector diffractometer using Mo Ka radiation $(\lambda=0.71073 \AA)$ at $\mathrm{T}=173$ (2) $\mathrm{K}$ (for 6) and $\mathrm{T}=296(2) \mathrm{K}$ (for 3 and 12). Absorption corrections by multi-scan ${ }^{30}$ were applied. Structures were solved by direct methods and refined by fullmatrix least squares against $\mathrm{F}^{2}$ using all data. ${ }^{31,32}$ All non- $\mathrm{H}$ atoms were refined anisotropically. Aromatic, metyhylene and methyl $\mathrm{H}$ atoms were positioned geometrically at distances of 0.93 (aromatic $\mathrm{CH}), 0.97\left(\mathrm{CH}_{2}\right)$ and $0.96\left(\mathrm{CH}_{3}\right)$ from the parent $\mathrm{C}$ atoms; a riding model was used during the refinement process and the $\operatorname{Uiso}(\mathrm{H})$ values were constrained to be $1.2 \mathrm{Ueq}$ (for aromatic and methylene carrier atoms) and $1.5 \mathrm{Ueq}$ (for methyl carrier atoms). Crystallographic data of 6 are; Empirical formula : $\mathrm{C}_{24} \mathrm{H}_{38} \mathrm{Cl}_{2} \mathrm{FeN}_{7} \mathrm{P}_{3}$, formula weight: 644.27, crystal system : monoclinic, space group : C2/c, $a(\AA)$ : 27.5472(5), $b(\AA): 11.0280(3), c(\AA): 20.4061(4), \alpha\left(^{\circ}\right):$ 90.00, $\beta\left(^{\circ}\right): 90.00, \gamma\left(^{\circ}\right): 90.00, V\left(\AA^{3}\right): 5965.4(2), \mathrm{Z}: 8, \mu$ $\left(\mathrm{cm}^{-1}\right): 0.873\left(\mathrm{Mo} \mathrm{K}_{\alpha}\right), \rho$ (Calc. $)\left(\mathrm{g} \mathrm{cm}^{-3}\right): 1.435$, number of reflections total : 27883 , number of reflections unique: 5278 , $R_{\text {int }}: 0.0253,2 \theta_{\max }\left(^{\circ}\right): 50.06, T_{\min } / T_{\max }: 0.8765 / 0.9543$, number of parameters : $335, \mathrm{R}\left[\mathrm{F}^{2}>2 \sigma\left(\mathrm{F}^{2}\right)\right]: 0.0519$, wR : 0.1381 .

\subsection{Preparation of the compounds}

The tetrachloro NN and NO spirocyclic monoferrorrocenyl pendant-armed spirocyclotriphosphazenes (1-5) were obtained from the reactions of monoferrocenylamines with trimer according to the methods reported in the literature. ${ }^{3 c,} 33$ See Scheme 1 for structures.

2.5a Synthesis of 6: $0.31 \mathrm{~mL}$ of piperidine $(3.10 \mathrm{mmol})$ in THF $(25 \mathrm{~mL})$ was added to $\mathbf{1}(0.850 \mathrm{~g}, 1.55 \mathrm{mmol})$ and triethylamine $(0.87 \mathrm{~mL})$ in dry THF $(100 \mathrm{~mL})$ with stirring and refluxing for $10 \mathrm{~h}$. The reaction was followed on TLC silica gel plates using toluene-THF (7:1). After the solvent was evaporated, the product was purified by column chromatography with toluene-THF (7:1). Yield: $0.82 \mathrm{~g}$ (82\%), M.p. $162{ }^{\circ} \mathrm{C}$. Anal. Calc. for $\mathrm{C}_{24} \mathrm{H}_{38} \mathrm{~N}_{7} \mathrm{FeP}_{3} \mathrm{Cl}_{2}$ : C, 44.74; H, 5.94; N, 15.22. Found: C, 45.01; H, 5.86; N, 15.34. APIES-MS (fragments were based on ${ }^{35} \mathrm{Cl}$ and $\left.{ }^{56} \mathrm{Fe}, \mathrm{Ir} \%\right): m / z 644\left([\mathrm{MH}]^{+}\right.$, 100.0). FTIR (KBr, cm ${ }^{-1}$ ): 3079 (C-H arom.), 2960, 2854 (C-H aliph.), 1187 ( $\mathrm{P}=\mathrm{N}), 553$ (asymm.), 511 (symm.) ( $\mathrm{PCl}$ ). ${ }^{1} \mathrm{H} \mathrm{NMR}\left(\mathrm{CDCl}_{3}, \mathrm{ppm}\right.$, numberings of protons are given in Scheme 1): $\delta 1.56\left[\mathrm{~m}, 4 \mathrm{H}, \mathrm{NCH}_{2} \mathrm{CH}_{2} \mathrm{CH}_{2}\right.$ (pip)], $1.62[\mathrm{~m}$, $8 \mathrm{H}, \mathrm{NCH}_{2} \mathrm{CH}_{2}$ (pip)], 2.53 (d, $3 \mathrm{H},{ }^{3} \mathrm{~J}_{\mathrm{PH}}=12.0 \mathrm{~Hz}, \mathrm{CH}_{3}$ ), 2.98-3.18 (m, 2H, NCH $H_{2}$, 2.98-3.18 [m, 8H, $\mathrm{NCH}_{2}$ (pip)], 2.98-3.18 (m, 2H, $\left.\mathrm{CH}_{3} \mathrm{NCH}_{2}\right), 3.85\left(\mathrm{dd}, 1 \mathrm{H},{ }^{3} J_{\mathrm{PH}}=7.2 \mathrm{~Hz}\right.$, $\left.{ }^{2} J_{\mathrm{HH}}=13.8 \mathrm{~Hz}, \mathrm{H} 5\right), 3.91\left(\mathrm{dd}, 1 \mathrm{H},{ }^{3} J_{\mathrm{PH}}=7.0 \mathrm{~Hz}\right.$, $\left.{ }^{2} J_{\mathrm{HH}}=13.8 \mathrm{~Hz}, \mathrm{H} 5\right), 4.12(5 \mathrm{H}, \mathrm{H} 4), 4.09$ (2H, H3), 4.13 $(2 \mathrm{H}, \mathrm{H} 2) .{ }^{13} \mathrm{C} \mathrm{NMR}\left(\mathrm{CDCl}_{3}, \mathrm{ppm}\right.$, numberings of carbons are given in Scheme 1): $\delta 24.81\left[\mathrm{NCH}_{2} \mathrm{CH}_{2} \mathrm{CH}_{2}\right.$ (pip)], 24.96 $\left[\mathrm{NCH}_{2} \mathrm{CH}_{2} \mathrm{CH}_{2}\right.$ (pip)], $26.13\left[\mathrm{~d},{ }^{3} \mathrm{~J}_{\mathrm{PC}}=6.8 \mathrm{~Hz}, \mathrm{NCH}_{2} \mathrm{CH}_{2}\right.$ (pip)], $26.29\left[\mathrm{~d},{ }^{3} \mathrm{~J}_{\mathrm{PC}}=6.1 \mathrm{~Hz}, \mathrm{NCH}_{2} \mathrm{CH}_{2}\right.$ (pip)], 31.43 $\left(\mathrm{d},{ }^{2} J_{\mathrm{PC}}=3.9 \mathrm{~Hz}, C \mathrm{H}_{3}\right), 43.48\left(\mathrm{~d},{ }^{2} J_{\mathrm{PC}}=13.0 \mathrm{~Hz}\right.$, 
$\left.\mathrm{CH}_{3} \mathrm{NCH}_{2}\right), 44.31\left(\mathrm{~d},{ }^{2} J_{\mathrm{PC}}=6.1 \mathrm{~Hz}, \mathrm{C} 5\right), 45.12\left[\mathrm{NCH}_{2}\right.$ (pip)], $45.24\left[\mathrm{NCH}_{2}\right.$ (pip)], $47.06\left(\mathrm{~d},{ }^{2} J_{\mathrm{PC}}=12.2 \mathrm{~Hz}\right.$, $\left.\mathrm{NCH}_{2}\right), 68.41(\mathrm{C} 4), 68.25(\mathrm{C} 3), 69.89(\mathrm{C} 2), 83.72\left(\mathrm{~d},{ }^{3} J_{\mathrm{PC}}=\right.$ $11.8 \mathrm{~Hz}, \mathrm{C} 1)$.

2.5b Synthesis of 7: For the synthesis, the procedure used for 6 was followed; using $2(0.77 \mathrm{~g}, 1.37 \mathrm{mmol})$, piperidine $(0.27 \mathrm{~mL}, 2.74 \mathrm{mmol})$ and triethylamine $(0.77 \mathrm{~mL})$. Yield: $0.76 \mathrm{~g}(84 \%)$, M.p. $154^{\circ} \mathrm{C}$. Anal. Calc. for $\mathrm{C}_{25} \mathrm{H}_{40} \mathrm{~N}_{7} \mathrm{FeP}_{3} \mathrm{Cl}_{2}$ : C, 45.61; H, 6.12; N, 14.89. Found: C, 45.36; H, 6.43; N, 14.62. APIES-MS (fragments were based on ${ }^{35} \mathrm{Cl}$ and ${ }^{56} \mathrm{Fe}, \mathrm{Ir}$ $\%): m / z 658\left([\mathrm{MH}]^{+}, 100.0\right)$. FTIR $\left(\mathrm{KBr}, \mathrm{cm}^{-1}\right): 3078(\mathrm{C}-\mathrm{H}$ arom.), 2933, 2850 (C-H aliph.), $1170(\mathrm{P}=\mathrm{N}), 578$ (asymm.) 518 (symm.) ( $\mathrm{PCl}) .{ }^{1} \mathrm{H} \mathrm{NMR}\left(\mathrm{CDCl}_{3}, \mathrm{ppm}\right.$, numberings of protons are given in Scheme 1): $\delta 1.15\left(\mathrm{t}, 3 \mathrm{H},{ }^{3} J_{\mathrm{HH}}=\right.$ $7.2 \mathrm{~Hz}, \mathrm{CH}_{3}$ ), $1.55\left[\mathrm{~m}, 4 \mathrm{H}, \mathrm{NCH}_{2} \mathrm{CH}_{2} \mathrm{CH}_{2}\right.$ (pip)], 1.63 [m, 8H, $\mathrm{NCH}_{2} \mathrm{CH}_{2}$ (pip)], 2.86-3.06 (m, 2H, $\mathrm{NCH}_{2} \mathrm{CH}_{3}$ ), 2.86-3.06 (m, 2H, NCH $H_{2}$ ) 3.09 [m, 4H, NCH $H_{2}$ (pip)], 3.18 [m, 4H, NCH$H_{2}$ (pip)], 2.86-3.06 (m, 2H, ${ }^{3} J_{\mathrm{HH}}=7.2 \mathrm{~Hz}$, $\left.\mathrm{C}_{2} \mathrm{H}_{5} \mathrm{NCH}_{2}\right), 3.71\left(\mathrm{dd}, 1 \mathrm{H},{ }^{3} J_{\mathrm{PH}}=6.8 \mathrm{~Hz},{ }^{2} J_{\mathrm{HH}}=14.0 \mathrm{~Hz}\right.$, H5), $3.86\left(\mathrm{dd}, 1 \mathrm{H},{ }^{3} J_{\mathrm{PH}}=6.8 \mathrm{~Hz},{ }^{2} J_{\mathrm{HH}}=14.0 \mathrm{~Hz}, \mathrm{H} 5\right)$, $4.10(5 \mathrm{H}, \mathrm{H} 4), 4.09(1 \mathrm{H}, \mathrm{H} 3), 4.12(1 \mathrm{H}, \mathrm{H} 3), 4.34(2 \mathrm{H}$, $\mathrm{H} 2) .{ }^{13} \mathrm{C} \mathrm{NMR}\left(\mathrm{CDCl}_{3}, \mathrm{ppm}\right.$, numberings of carbons are given in Scheme 1): $\delta 13.81\left(\mathrm{~d},{ }^{3} J_{\mathrm{PC}}=5.3 \mathrm{~Hz}, C \mathrm{H}_{3}\right)$, $24.83\left[\mathrm{NCH}_{2} \mathrm{CH}_{2} \mathrm{CH}_{2}\right.$ (pip)], $24.98\left[\mathrm{NCH}_{2} \mathrm{CH}_{2} \mathrm{CH}_{2}\right.$ (pip)], $26.16\left[\mathrm{~d},{ }^{3} J_{\mathrm{PC}}=6.8 \mathrm{~Hz}, \mathrm{NCH}_{2} \mathrm{CH}_{2}\right.$ (pip)], 26.32 [d, ${ }^{3} J_{\mathrm{PC}}=6.9 \mathrm{~Hz}, \mathrm{NCH}_{2} \mathrm{CH}_{2}$ (pip)], $39.23\left(\mathrm{~d},{ }^{2} J_{\mathrm{PC}}=4.6 \mathrm{~Hz}\right.$, $\left.\mathrm{NCH}_{2} \mathrm{CH}_{3}\right), 43.52\left(\mathrm{~d},{ }^{2} J_{\mathrm{PC}}=13.0 \mathrm{~Hz}, \mathrm{NCH}_{2}\right), 43.65(\mathrm{~d}$, $\left.{ }^{2} J_{\mathrm{PC}}=12.9 \mathrm{~Hz}, \mathrm{C}_{2} \mathrm{H}_{5} \mathrm{NCH}_{2}\right), 44.28\left(\mathrm{~d},{ }^{2} J_{\mathrm{PC}}=6.1 \mathrm{~Hz}\right.$, C5), $45.28\left[\mathrm{~d},{ }^{2} J_{\mathrm{PC}}=2.3 \mathrm{~Hz}, \mathrm{NCH}_{2}\right.$ (pip)], $45.95[\mathrm{~d}$, ${ }^{2} J_{\mathrm{PC}}=2.5 \mathrm{~Hz}, \mathrm{NCH}_{2}$ (pip)], 68.41 (C4), 68.00 (C3), 68.38 (C3), 69.34 (C2), $69.94(\mathrm{C} 2), 83.50\left(\mathrm{~d},{ }^{3} J_{\mathrm{PC}}=9.2 \mathrm{~Hz}, \mathrm{C} 1\right)$.

2.5c Synthesis of 8 : The work-up procedure was similar to that of compound 6, using $3(0.72 \mathrm{~g}, 1.28 \mathrm{mmol})$, piperidine $(0.25 \mathrm{~mL}, 1.56 \mathrm{mmol})$ and triethylamine (0.44 mL). Yield: 0.66 g (78\%), M.p. $101^{\circ}$ C. Anal. Calc. for $\mathrm{C}_{25} \mathrm{H}_{40} \mathrm{~N}_{7} \mathrm{FeP}_{3} \mathrm{Cl}_{2}$ : C, 45.61; H, 6.12; N, 14.89. Found: C, 45.28; H, 6.33; N, 15.03. APIES-MS (fragments were based on ${ }^{35} \mathrm{Cl}$ and ${ }^{56} \mathrm{Fe}$, Ir \%): $m / z 658\left([\mathrm{MH}]^{+}, 100.0\right)$. FTIR ( $\left.\mathrm{KBr}, \mathrm{cm}^{-1}\right)$ : 3089 (C-H arom.), 2956, 2879 (C-H aliph.), $1197(\mathrm{P}=\mathrm{N}), 561$ (asymm.) 512 (symm.) ( $\mathrm{PCl}) .{ }^{1} \mathrm{H}$ NMR $\left(\mathrm{CDCl}_{3}, \mathrm{ppm}\right.$, numberings of protons are given in Scheme 1): $\delta 1.53\left[\mathrm{~m}, 4 \mathrm{H},{ }^{3} J_{\mathrm{HH}}=5.0 \mathrm{~Hz}, \mathrm{NCH}_{2} \mathrm{CH}_{2} \mathrm{CH}_{2}\right.$ (pip)], $1.58\left[\mathrm{~m}, 8 \mathrm{H},{ }^{3} J_{\mathrm{HH}}=5.7 \mathrm{~Hz}, \mathrm{NCH}_{2} \mathrm{CH}_{2}\right.$ (pip)], $1.66[\mathrm{~m}$, $2 \mathrm{H}, \mathrm{NCH}_{2} \mathrm{CH}_{2}$ (spiro)], $2.52\left(\mathrm{~d}, 3 \mathrm{H},{ }^{3} \mathrm{~J}_{\mathrm{PH}}=12.4 \mathrm{~Hz}, \mathrm{CH}_{3}\right)$, $2.89\left(\mathrm{~m}, 2 \mathrm{H}, \mathrm{CH}_{3} \mathrm{NCH}_{2}\right), 2.92\left(\mathrm{~m}, 2 \mathrm{H}, \mathrm{NCH}_{2}\right), 3.12[\mathrm{~m}$, $4 \mathrm{H}, \mathrm{NCH}_{2}$ (pip)], 3.16 [m, 4H, $\mathrm{NCH}_{2}$ (pip)], 3.74 (dd, 1H, $\left.{ }^{3} J_{\mathrm{PH}}=5.8 \mathrm{~Hz},{ }^{2} J_{\mathrm{HH}}=13.6 \mathrm{~Hz}, \mathrm{H} 5\right), 3.88(\mathrm{dd}, 1 \mathrm{H}$, $\left.{ }^{3} J_{\mathrm{PH}}=6.0 \mathrm{~Hz},{ }^{2} J_{\mathrm{HH}}=13.6 \mathrm{~Hz}, \mathrm{H} 5\right), 4.13(5 \mathrm{H}, \mathrm{H} 4), 4.10$ $(1 \mathrm{H}, \mathrm{H} 3), 4.22(2 \mathrm{H}, \mathrm{H} 2) .{ }^{13} \mathrm{C} \mathrm{NMR}\left(\mathrm{CDCl}_{3}\right.$, ppm, numberings of carbons are given in Scheme 1): $\delta 24.46\left(\mathrm{NCH}_{2} \mathrm{CH}_{2}\right)$, $24.94\left[\mathrm{NCH}_{2} \mathrm{CH}_{2} \mathrm{CH}_{2}\right.$ (pip)], 25.06 [ $\mathrm{NCH}_{2} \mathrm{CH}_{2} \mathrm{CH}_{2}$ (pip)], $26.82\left[\mathrm{~d},{ }^{3} J_{\mathrm{PC}}=9.1 \mathrm{~Hz}, \mathrm{NCH}_{2} \mathrm{CH}_{2}\right.$ (pip)], $26.94\left[\mathrm{~d},{ }^{3} \mathrm{~J}_{\mathrm{PC}}=\right.$ $9.0 \mathrm{~Hz}, \mathrm{NCH}_{2} \mathrm{CH}_{2}$ (pip)], $36.37\left(\mathrm{CH}_{3}\right), 44.95\left(\mathrm{CH}_{3} \mathrm{NCH}_{2}\right)$, $45.42\left[\mathrm{NCH}_{2}\right.$ (pip)], 45.67 [ $\mathrm{NCH}_{2}$ (pip)], 46.09 (C5), 49.95 $\left(\mathrm{NCH}_{2}\right), 68.52$ (C4), 68.39 (C3), 68.45 (C3), 69.88 (C2), $70.02(\mathrm{C} 2), 84.93\left(\mathrm{~d},{ }^{3} J_{\mathrm{PC}}=11.6 \mathrm{~Hz}, \mathrm{C} 1\right)$. 2.5d Synthesis of 9: The procedure used for 6 was followed; using $4(0.85 \mathrm{~g}, 1.59 \mathrm{mmol})$, piperidine $(0.31 \mathrm{~mL}$, $3.18 \mathrm{mmol})$ and triethylamine $(0.90 \mathrm{~mL})$. Yield: $0.83 \mathrm{~g}$ (83\%), M.p. $108{ }^{\circ}$ C. Anal. Calc. for $\mathrm{C}_{23} \mathrm{H}_{35} \mathrm{~N}_{6} \mathrm{OFeP}_{3} \mathrm{Cl}_{2}$ : C, 43.76; H, 5.59; N, 13.31. Found: C, 43.95; H, 5.67; N, 13.24. APIES-MS (fragments were based on ${ }^{35} \mathrm{Cl}$ and ${ }^{56} \mathrm{Fe}$, Ir \%): $m / z 31\left([\mathrm{MH}]^{+}, 100.0\right)$. FTIR $\left(\mathrm{KBr}, \mathrm{cm}^{-1}\right): 3079$ (C-H arom.), 2982, 2853 (C-H aliph.), $1192(\mathrm{P}=\mathrm{N}), 572$ (asymm.), 528 (symm.) (PCl). ${ }^{1} \mathrm{H} \mathrm{NMR}\left(\mathrm{CDCl}_{3}\right.$, ppm, numberings of protons are given in Scheme 1): $\delta 1.54[\mathrm{~m}, 4 \mathrm{H}$, $\mathrm{NCH}_{2} \mathrm{CH}_{2} \mathrm{CH}_{2}$ (pip)], 1.62 [m, 8H, $\mathrm{NCH}_{2} \mathrm{CH}_{2}$ (pip)], 2.86$3.06\left(\mathrm{~m}, 2 \mathrm{H},{ }^{3} J_{\mathrm{HH}}=4.8 \mathrm{~Hz}, \mathrm{NCH}_{2}\right), 3.04\left[\mathrm{~m}, 4 \mathrm{H}, \mathrm{NCH}_{2}\right.$ (pip)], 3.15 [m, 4H, $\mathrm{NCH}_{2}$ (pip)], 3.75 (dd, $1 \mathrm{H},{ }^{3} J_{\mathrm{PH}}=$ $\left.7.2 \mathrm{~Hz},{ }^{2} J_{\mathrm{HH}}=13.4 \mathrm{~Hz}, \mathrm{H} 5\right), 3.88\left(\mathrm{dd}, 1 \mathrm{H},{ }^{3} J_{\mathrm{PH}}=6.8 \mathrm{~Hz}\right.$, $\left.{ }^{2} J_{\mathrm{HH}}=13.4 \mathrm{~Hz}, \mathrm{H} 5\right), 4.11(5 \mathrm{H}, \mathrm{H} 4), 4.13(2 \mathrm{H}, \mathrm{H} 3), 4.20$ $\left(\mathrm{m}, 2 \mathrm{H},{ }^{3} J_{\mathrm{PH}}=6.8 \mathrm{~Hz},{ }^{3} J_{\mathrm{HH}}=4.8 \mathrm{~Hz}, \mathrm{OCH}_{2}\right), 4.33$ $(2 \mathrm{H}, \mathrm{H} 2) .{ }^{13} \mathrm{C} \mathrm{NMR}\left(\mathrm{CDCl}_{3}\right.$, ppm, numberings of carbons are given in Scheme 1): $\delta 24.78\left[\mathrm{NCH}_{2} \mathrm{CH}_{2} \mathrm{CH}_{2}\right.$ (pip)], 24.93 $\left[\mathrm{NCH}_{2} \mathrm{CH}_{2} \mathrm{CH}_{2}\right.$ (pip)], $25.99\left[\mathrm{~d},{ }^{3} \mathrm{~J}_{\mathrm{PC}}=6.5 \mathrm{~Hz}, \mathrm{NCH}_{2} \mathrm{CH}_{2}\right.$ (pip)], 26.25 [d, ${ }^{3} J_{\mathrm{PC}}=6.4 \mathrm{~Hz}, \mathrm{NCH}_{2} \mathrm{CH}_{2}$ (pip)], 44.06 $\left(\mathrm{d},{ }^{2} J_{\mathrm{PC}}=5.7 \mathrm{~Hz}, \mathrm{C} 5\right), 45.51\left[\mathrm{NCH}_{2}\right.$ (pip)], $45.71\left[\mathrm{NCH}_{2}\right.$ (pip)], $45.56\left(\mathrm{~d},{ }^{2} J_{\mathrm{PC}}=14.8 \mathrm{~Hz}, \mathrm{NCH} \mathrm{H}_{2}\right), 64.29\left(\mathrm{OCH}_{2}\right)$, 68.67 (C4), 68.37 (C3), 68.56 (C3), 69.46 (C2), 69.93 (C2), $83.20\left(\mathrm{~d},{ }^{3} J_{\mathrm{PC}}=10.3 \mathrm{~Hz}, \mathrm{C} 1\right)$.

2.5e Synthesis of 10: For the synthesis, the procedure used for 6 was followed; using 5 (0.65 g, $1.19 \mathrm{mmol})$, piperidine $(0.23 \mathrm{~mL}, 2.38 \mathrm{mmol})$ and triethylamine $(0.67 \mathrm{~mL})$. The first product eluted was the mono-DASD-substituted derivative (5a). Yield: $0.61 \mathrm{~g}(79 \%)$, M.p. $162{ }^{\circ} \mathrm{C}$. Anal. Calc. for $\mathrm{C}_{24} \mathrm{H}_{37} \mathrm{~N}_{6} \mathrm{OFeP}_{3} \mathrm{Cl}_{2}$ : C, 44.67; H, 5.78; N, 13.02. Found: $\mathrm{C}$, 44.89; H, 5.77; N, 12.85. APIES-MS (fragments were based on ${ }^{35} \mathrm{Cl}$ and ${ }^{56} \mathrm{Fe}$, Ir \%): $m / z 644\left([\mathrm{M}]^{+}, 100.0\right)$. FTIR (KBr, $\mathrm{cm}^{-1}$ ): 3092 (C-H arom.), 2952, 2844 (C-H aliph.), 1186 $(\mathrm{P}=\mathrm{N}), 548$ (asymm.), 521 (symm.) ( $\mathrm{PCl}) .{ }^{1} \mathrm{H} \mathrm{NMR}\left(\mathrm{CDCl}_{3}\right.$, ppm, numberings of protons are given in Scheme 1): $1.54[\mathrm{~m}$, $4 \mathrm{H}, \mathrm{NCH}_{2} \mathrm{CH}_{2} \mathrm{CH}_{2}$ (pip)], 1.62 [m, 8H, $\mathrm{NCH}_{2} \mathrm{CH}_{2}$ (pip)], $1.93\left[\mathrm{~m}, 2 \mathrm{H},{ }^{3} \mathrm{~J}_{\mathrm{HH}}=6.0 \mathrm{~Hz}, \mathrm{NCH}_{2} \mathrm{CH}_{2}\right.$ (spiro)], 2.92-3.22 (m, 2H, $\mathrm{NCH}_{2}$ ), 2.92-3.22 [m, 8H, $\mathrm{NCH}_{2}$ (pip)], 3.68 (dd, $\left.1 \mathrm{H},{ }^{3} J_{\mathrm{PH}}=6.0 \mathrm{~Hz},{ }^{2} J_{\mathrm{HH}}=13.2 \mathrm{~Hz}, \mathrm{H} 5\right), 3.93(\mathrm{dd}, 1 \mathrm{H}$, $\left.{ }^{3} J_{\mathrm{PH}}=5.6 \mathrm{~Hz},{ }^{2} J_{\mathrm{HH}}=13.2 \mathrm{~Hz}, \mathrm{H} 5\right), 4.09(5 \mathrm{H}, \mathrm{H} 4), 4.11$ (1H, H3), 4.12 (1H, H3), 4.21 (1H, H2), 4.25 (1H, H2), 4.31 $\left(\mathrm{m}, 2 \mathrm{H},{ }^{3} J_{\mathrm{PH}}=6.8 \mathrm{~Hz},{ }^{3} J_{\mathrm{HH}}=6.0 \mathrm{~Hz}, \mathrm{OCH}_{2}\right) .{ }^{13} \mathrm{C} \mathrm{NMR}$ $\left(\mathrm{CDCl}_{3}, \mathrm{ppm}\right.$, numberings of carbons are given in Scheme 1): $\delta 24.83\left[\mathrm{NCH}_{2} \mathrm{CH}_{2} \mathrm{CH}_{2}\right.$ (pip)], $24.96\left[\mathrm{NCH}_{2} \mathrm{CH}_{2} \mathrm{CH}_{2}\right.$ (pip)], $26.01\left[\mathrm{~d},{ }^{3} J_{\mathrm{PC}}=6.4 \mathrm{~Hz}, \mathrm{NCH}_{2} \mathrm{CH}_{2}\right.$ (pip)], 26.29 [d, ${ }^{3} J_{\mathrm{PC}}=7.1 \mathrm{~Hz}, \mathrm{NCH}_{2} \mathrm{CH}_{2}$ (pip)], 26.09 (d, ${ }^{3} J_{\mathrm{PC}}=3.8 \mathrm{~Hz}$, $\mathrm{NCH}_{2} \mathrm{CH}_{2}$ ), 45.04 [ $\mathrm{NCH}_{2}$ (pip)], 45.18 [ $\mathrm{NCH}_{2}$ (pip)], 45.06 $\left(\mathrm{NCH}_{2}\right), 47.24(\mathrm{C} 5), 67.11\left(\mathrm{~d},{ }^{2} J_{\mathrm{PC}}=7.1 \mathrm{~Hz}, \mathrm{OCH}_{2}\right), 68.44$ (C4), 68.12 (C3), 68.36 (C3), 69.82 (C2), 70.60 (C2), 82.59 $\left(\mathrm{d},{ }^{3} J_{\mathrm{PC}}=11.5 \mathrm{~Hz}, \mathrm{C} 1\right)$.

2.5f Synthesis of 11: A solution of piperidine $(1.45 \mathrm{~mL}$, $14.63 \mathrm{mmol})$ in dry THF (25 mL) was added to a solution of $1(1.00 \mathrm{~g}, 1.83 \mathrm{mmol})$ and triethylamine $(0.72 \mathrm{~mL})$ in dry THF $(100 \mathrm{~mL})$ with stirring and refluxing for $18 \mathrm{~h}$. After the solvent was evaporated, the product was purified by column 
chromatography with toluene-THF (2:1). The first product eluted was the geminal-piperidino-substituted derivative (6). Yield: $0.25 \mathrm{~g}(21 \%)$. The second product was the tetrakispiperidino-substituted derivative (11). Yield: $0.96 \mathrm{~g}(71 \%)$, M.p. $160{ }^{\circ}$ C. Anal. Calc. for $\mathrm{C}_{34} \mathrm{H}_{58} \mathrm{~N}_{9} \mathrm{FeP}_{3}$ : C, 55.06; H, 7.88; N, 17.00. Found: C, 54.75; H, 7.51; N, 17.36. APIESMS (fragments were based on $\left.{ }^{56} \mathrm{Fe}, \mathrm{Ir} \%\right): \mathrm{m} / z 742\left([\mathrm{MH}]^{+}\right.$, 100.0). FTIR (KBr, cm ${ }^{-1}$ ): 3072 (C-H arom.), 2929, 2848 (C-H aliph.), $1195(\mathrm{P}=\mathrm{N}) .{ }^{1} \mathrm{H} \mathrm{NMR}\left(\mathrm{CDCl}_{3}, \mathrm{ppm}\right.$, numberings of protons are given in Scheme 1): $\delta 1.51[\mathrm{~m}, 8 \mathrm{H}$, $\mathrm{NCH}_{2} \mathrm{CH}_{2} \mathrm{CH}_{2}$ (pip)], 1.58 [m, 16H, $\mathrm{NCH}_{2} \mathrm{CH}_{2}$ (pip)], 2.51 $\left(\mathrm{d}, 3 \mathrm{H},{ }^{3} J_{\mathrm{PH}}=11.2 \mathrm{~Hz}, \mathrm{CH}_{3}\right), 2.93\left(\mathrm{~m}, 2 \mathrm{H},{ }^{3} J_{\mathrm{PH}}=10.4 \mathrm{~Hz}\right.$, $\left.{ }^{3} J_{\mathrm{HH}}=6.4 \mathrm{~Hz}, \mathrm{CH}_{3} \mathrm{NCH}_{2}\right), 3.09\left(\mathrm{~m}, 2 \mathrm{H},{ }^{3} J_{\mathrm{PH}}=10.4 \mathrm{~Hz}\right.$, ${ }^{3} J_{\mathrm{HH}}=6.4 \mathrm{~Hz}, \mathrm{NCH}_{2}$ ), 3.07 [m, 8H, NCH (pip)], 3.16 $\left[\mathrm{m}, 8 \mathrm{H}, \mathrm{NCH}_{2}\right.$ (pip)], $3.72\left(\mathrm{~d}, 2 \mathrm{H},{ }^{3} J_{\mathrm{PH}}=5.2 \mathrm{~Hz}, \mathrm{H} 5\right)$, $4.09\left(\mathrm{~m}, 5 \mathrm{H},{ }^{4} J_{\mathrm{HH}}=1.8 \mathrm{~Hz},{ }^{4} J_{\mathrm{HH}}=1.8 \mathrm{~Hz}, \mathrm{H} 4\right), 4.06$ $\left(\mathrm{m}, 2 \mathrm{H},{ }^{3} J_{\mathrm{HH}}=3.6 \mathrm{~Hz},{ }^{4} J_{\mathrm{HH}}=1.8 \mathrm{~Hz}, \mathrm{H} 3\right), 4.15(\mathrm{~m}, 2 \mathrm{H}$, $\left.{ }^{3} J_{\mathrm{HH}}=3.6 \mathrm{~Hz},{ }^{4} J_{\mathrm{HH}}=1.8 \mathrm{~Hz}, \mathrm{H} 2\right) .{ }^{13} \mathrm{C} \mathrm{NMR}\left(\mathrm{CDCl}_{3}\right.$, ppm, numberings of carbons are given in Scheme 1): $\delta 25.33$ [ $\mathrm{NCH}_{2} \mathrm{CH}_{2} \mathrm{CH}_{2}$ (pip)], 25.47 [ $\mathrm{NCH}_{2} \mathrm{CH}_{2} \mathrm{CH}_{2}$ (pip)], 26.69 $\left[\mathrm{d},{ }^{3} J_{\mathrm{PC}}=8.4 \mathrm{~Hz}, \mathrm{NCH}_{2} \mathrm{CH}_{2}\right.$ (pip)], $26.77\left[\mathrm{~d},{ }^{3} J_{\mathrm{PC}}=\right.$ $6.7 \mathrm{~Hz}, \mathrm{NCH}_{2} \mathrm{CH}_{2}$ (pip)], $32.38\left(\mathrm{~d},{ }^{2} \mathrm{~J}_{\mathrm{PC}}=3.8 \mathrm{~Hz}, \mathrm{CH}_{3}\right.$ ), $44.35\left(\mathrm{~d},{ }^{2} J_{\mathrm{PC}}=10.7 \mathrm{~Hz}, \mathrm{CH}_{3} \mathrm{NCH}_{2}\right), 45.62\left[\mathrm{NCH}_{2}\right.$ (pip)], $45.74\left[\mathrm{NCH}_{2}\right.$ (pip)], 45.95 (d, $\left.{ }^{2} J_{\mathrm{PC}}=4.5 \mathrm{~Hz}, \mathrm{C} 5\right), 47.29$ (d, $\left.{ }^{2} J_{\mathrm{PC}}=12.0 \mathrm{~Hz}, \mathrm{NCH}_{2}\right), 68.52(\mathrm{C} 4), 68.10$ (C3), $69.84(\mathrm{C} 2)$, $85.03\left(\mathrm{~d},{ }^{3} J_{\mathrm{PC}}=12.2 \mathrm{~Hz}, \mathrm{C} 1\right)$.

$2.5 \mathrm{~g}$ Synthesis of 12: For the preparation of $\mathbf{1 2}$ the procedure used for 11 was followed; using $2(0.70 \mathrm{~g}, 1.25 \mathrm{mmol})$, piperidine $(0.99 \mathrm{~mL}, 10.00 \mathrm{mmol})$ and triethylamine $(1.41 \mathrm{~mL})$. The first product eluted was the geminal-piperidino-substituted derivative (7). Yield: $0.15 \mathrm{~g}(18 \%)$. The second product was the tetrakis-piperidino-substituted derivative (12). Yield: 0.71 g (75\%), M.p. $186{ }^{\circ}$ C. Anal. Calc. for $\mathrm{C}_{35} \mathrm{H}_{60} \mathrm{~N}_{9} \mathrm{FeP}_{3}$ : C, 55.63; H, 8.00; N, 16.68. Found: C, 55.63; H, 8.30; N, 16.45. APIES-MS (fragments were based on ${ }^{56} \mathrm{Fe}, \mathrm{Ir} \%$ ): $\mathrm{m} / \mathrm{z} 756$ $\left([\mathrm{M}]^{+}, 100.0\right)$. FTIR (KBr, $\left.\mathrm{cm}^{-1}\right): 3087$ (C-H arom.), 2954, 2840 (C-H aliph.), 1193 (P=N). ${ }^{1} \mathrm{H} \mathrm{NMR}\left(\mathrm{CDCl}_{3}\right.$, ppm, numberings of protons are given in Scheme 1): $\delta 1.10(\mathrm{t}, 3 \mathrm{H}$, $\left.{ }^{3} J_{\mathrm{HH}}=7.1 \mathrm{~Hz}, \mathrm{CH}_{3}\right), 1.51\left[\mathrm{~m}, 8 \mathrm{H}, \mathrm{NCH}_{2} \mathrm{CH}_{2} \mathrm{CH}_{2}\right.$ (pip)], $1.58\left[\mathrm{~m}, 16 \mathrm{H}, \mathrm{NCH}_{2} \mathrm{CH}_{2}\right.$ (pip)], $2.86\left(\mathrm{~m}, 2 \mathrm{H},{ }^{3} J_{\mathrm{HH}}=7.1 \mathrm{~Hz}\right.$, $\left.\mathrm{NCH}_{2} \mathrm{CH}_{3}\right), 2.93\left(\mathrm{~m}, 2 \mathrm{H},{ }^{3} J_{\mathrm{HH}}=6.4 \mathrm{~Hz},{ }^{3} J_{\mathrm{PH}}=10.0 \mathrm{~Hz}\right.$, $\left.\mathrm{C}_{2} \mathrm{H}_{5} \mathrm{NCH}_{2}\right), 3.00\left(\mathrm{~m}, 2 \mathrm{H},{ }^{3} J_{\mathrm{HH}}=6.42 \mathrm{~Hz}, \mathrm{NCH}_{2}\right), 3.06$ $\left[\mathrm{m}, 8 \mathrm{H}, \mathrm{NCH}_{2}\right.$ (pip)], $3.15\left[\mathrm{~m}, 8 \mathrm{H}, \mathrm{NCH}_{2}\right.$ (pip)], 3.73 (d, $\left.2 \mathrm{H},{ }^{3} J_{\mathrm{PH}}=4.8 \mathrm{~Hz}, \mathrm{H} 5\right), 4.09(5 \mathrm{H}, \mathrm{H} 4), 4.06(1 \mathrm{H}, \mathrm{H} 3)$, $4.15(2 \mathrm{H}, \mathrm{H} 2) .{ }^{13} \mathrm{C} \mathrm{NMR}\left(\mathrm{CDCl}_{3}\right.$, ppm, numberings of carbons are given in Scheme 1): $\delta 14.39\left(\mathrm{~d},{ }^{3} J_{\mathrm{PC}}=7.6 \mathrm{~Hz}\right.$, $\mathrm{CH}_{3}$ ), $25.32\left[\mathrm{NCH}_{2} \mathrm{CH}_{2} \mathrm{CH}_{2}\right.$ (pip)], $25.46\left[\mathrm{NCH}_{2} \mathrm{CH}_{2} \mathrm{CH}_{2}\right.$ (pip)], $26.67\left[\mathrm{~d},{ }^{3} J_{\mathrm{PC}}=6.6 \mathrm{~Hz}, \mathrm{NCH}_{2} \mathrm{CH}_{2}\right.$ (pip)], 26.77 [d, ${ }^{3} J_{\mathrm{PC}}=6.7 \mathrm{~Hz}, \mathrm{NCH}_{2} \mathrm{CH}_{2}$ (pip)], $39.88\left(\mathrm{~d},{ }^{2} J_{\mathrm{PC}}=5.0 \mathrm{~Hz}\right.$, $\left.\mathrm{NCH}_{2} \mathrm{CH}_{3}\right), 43.70\left(\mathrm{~d},{ }^{2} J_{\mathrm{PC}}=12.2 \mathrm{~Hz}, \mathrm{NCH}_{2}\right), 44.38(\mathrm{~d}$, $\left.{ }^{2} J_{\mathrm{PC}}=11.5 \mathrm{~Hz}, \mathrm{C}_{2} \mathrm{H}_{5} \mathrm{NCH}_{2}\right), 45.92\left(\mathrm{~d},{ }^{2} J_{\mathrm{PC}}=5.8 \mathrm{~Hz}\right.$, C5), 45.60 [ $\mathrm{NCH}_{2}$ (pip)], 45.77 [ $\mathrm{NCH}_{2}$ (pip)], 68.52 (C4), 68.09 (C3), 69.91 (C2), 84.99 (d, $\left.{ }^{3} J_{\mathrm{PC}}=12.9 \mathrm{~Hz}, \mathrm{C} 1\right)$.

2.5h Synthesis of 13: The procedure used for 11 was followed; using $3(0.70 \mathrm{~g}, 1.25 \mathrm{mmol})$, piperidine $(0.99 \mathrm{~mL}$, $10.00 \mathrm{mmol})$ and triethylamine $(1.41 \mathrm{~mL})$. The first product eluted was the geminal-piperidino-substituted derivative (8). Yield: $0.16 \mathrm{~g}(16 \%)$. The second product was the tetrakispiperidino-substituted derivative (13). Yield: $0.69 \mathrm{~g}(73 \%)$, M.p. $191{ }^{\circ} \mathrm{C}$. Anal. Calc. for $\mathrm{C}_{35} \mathrm{H}_{60} \mathrm{~N}_{9} \mathrm{FeP}_{3}$ : C, 55.63; H, 8.00; N, 16.68. Found: C, 55.79; H, 8.09; N, 16.35. APIESMS (fragments were based on $\left.{ }^{56} \mathrm{Fe}, \mathrm{Ir} \%\right): m / z 756\left([\mathrm{MH}]^{+}\right.$, 100.0). FTIR (KBr, cm ${ }^{-1}$ ): 3087 (C-H arom.), 2929, 2819 (C-H aliph.), $1193(\mathrm{P}=\mathrm{N}) .{ }^{1} \mathrm{H} \mathrm{NMR}\left(\mathrm{CDCl}_{3}, \mathrm{ppm}\right.$, numberings of protons are given in Scheme 1): $\delta 1.50[\mathrm{~m}$, $8 \mathrm{H},{ }^{3} J_{\mathrm{HH}}=5.6 \mathrm{~Hz}, \mathrm{NCH}_{2} \mathrm{CH}_{2} \mathrm{CH}_{2}$ (pip)], $1.55[\mathrm{~m}, 16 \mathrm{H}$, ${ }^{3} J_{\mathrm{HH}}=5.6 \mathrm{~Hz}, \mathrm{NCH}_{2} \mathrm{CH}_{2}$ (pip)], $1.65\left[\mathrm{~m}, 2 \mathrm{H}, \mathrm{NCH}_{2} \mathrm{CH}_{2}\right.$ (spiro)], $2.50\left(\mathrm{~d}, 3 \mathrm{H},{ }^{3} J_{\mathrm{PH}}=12.8 \mathrm{~Hz}, \mathrm{CH}_{3}\right), 2.93(\mathrm{~m}, 2 \mathrm{H}$, $\mathrm{CH}_{3} \mathrm{NCH}_{2}$ ), 2.97 (m, 2H, $\mathrm{NCH}_{2}$ ), 3.10 [m, 8H, $\mathrm{NCH}_{2}$ (pip)], $3.13\left[\mathrm{~m}, 8 \mathrm{H}, \mathrm{NCH}_{2}\right.$ (pip)], $3.77\left(\mathrm{~d}, 2 \mathrm{H},{ }^{3} J_{\mathrm{PH}}=5.6 \mathrm{~Hz}\right.$, $\mathrm{H} 5), 4.09\left(\mathrm{~m}, 5 \mathrm{H},{ }^{4} J_{\mathrm{HH}}=1.6 \mathrm{~Hz},{ }^{4} J_{\mathrm{HH}}=1.2 \mathrm{~Hz}, \mathrm{H} 4\right)$, $4.10\left(\mathrm{~m}, 1 \mathrm{H},{ }^{3} J_{\mathrm{HH}}=2.0 \mathrm{~Hz},{ }^{4} J_{\mathrm{HH}}=1.6 \mathrm{~Hz}, \mathrm{H} 3\right)$, $4.22\left(\mathrm{~m}, 2 \mathrm{H},{ }^{3} J_{\mathrm{HH}}=2.0 \mathrm{~Hz},{ }^{4} J_{\mathrm{HH}}=1.2 \mathrm{~Hz}, \mathrm{H} 2\right)$. ${ }^{13} \mathrm{C} \mathrm{NMR}\left(\mathrm{CDCl}_{3}, \mathrm{ppm}\right.$, numberings of carbons are given in Scheme 1): $\delta 24.33\left(\mathrm{NCH}_{2} \mathrm{CH}_{2}\right), 25.14\left[\mathrm{NCH}_{2} \mathrm{CH}_{2} \mathrm{CH}_{2}\right.$ (pip)], $25.23\left[\mathrm{NCH}_{2} \mathrm{CH}_{2} \mathrm{CH}_{2}\right.$ (pip)], $26.48\left[\mathrm{~d},{ }^{3} \mathrm{~J}_{\mathrm{PC}}=9.2 \mathrm{~Hz}\right.$, $\mathrm{NCH}_{2} \mathrm{CH}_{2}$ (pip)], 26.51 [d, ${ }^{3} \mathrm{~J}_{\mathrm{PC}}=9.2 \mathrm{~Hz}, \mathrm{NCH}_{2} \mathrm{CH}_{2}$ (pip)], $36.46\left(\mathrm{CH}_{3}\right), 44.96\left(\mathrm{CH}_{3} \mathrm{NCH}_{2}\right), 45.56\left[\mathrm{NCH}_{2}\right.$ (pip)], $45.59\left[\mathrm{NCH}_{2}\right.$ (pip)], 46.17 (C5), $50.88\left(\mathrm{NCH}_{2}\right), 68.29$ (C4), 68.39 (C3), 70.14 (C2), 85.07 (d, $\left.{ }^{3} J_{\mathrm{PC}}=11.9 \mathrm{~Hz}, \mathrm{C} 1\right)$.

2.5i Synthesis of 14: The work-up procedure was similar to that of compound 11, using $4(0.90 \mathrm{~g}, 1.69 \mathrm{mmol})$, piperidine $(1.34 \mathrm{~mL}, 13.52 \mathrm{mmol})$ and triethylamine $(1.90 \mathrm{~mL})$. The first product eluted was the geminal-piperidino-substituted derivative (9). Yield: $0.17 \mathrm{~g}(16 \%)$. The second product was the tetrakis-piperidino-substituted derivative (14). Yield: 0.69 g (73\%), M.p. $204{ }^{\circ}$ C. Anal. Calc. for $\mathrm{C}_{33} \mathrm{H}_{55} \mathrm{~N}_{8} \mathrm{OFeP}_{3}$ : C, 54.40; H, 7.61; N, 15.38. Found: C, 54.59; H, 7.40; N, 15.07. APIES-MS (fragments were based on ${ }^{56} \mathrm{Fe}, \mathrm{Ir} \%$ ): $m / z 729$ $\left([\mathrm{MH}]^{+}, 100.0\right)$. FTIR $\left(\mathrm{KBr}, \mathrm{cm}^{-1}\right): 3070$ (C-H arom.), 2929, 2848 (C-H aliph.), $1191(\mathrm{P}=\mathrm{N}) .{ }^{1} \mathrm{H} \mathrm{NMR}\left(\mathrm{CDCl}_{3}, \mathrm{ppm}\right.$, numberings of protons are given in Scheme 1): $\delta 1.51[\mathrm{~m}, 8 \mathrm{H}$, $\mathrm{NCH}_{2} \mathrm{CH}_{2} \mathrm{CH}_{2}$ (pip)], 1.60 [m, 16H, $\mathrm{NCH}_{2} \mathrm{CH}_{2}$ (pip)], 3.02$3.12\left(\mathrm{~m}, 2 \mathrm{H},{ }^{3} J_{\mathrm{HH}}=6.4 \mathrm{~Hz}, \mathrm{NCH}_{2}\right), 3.18\left[\mathrm{~m}, 16 \mathrm{H}, \mathrm{NCH}_{2}\right.$ (pip)], 3.80 (d, 2H, $\left.{ }^{3} J_{\mathrm{PH}}=5.2 \mathrm{~Hz}, \mathrm{H} 5\right), 4.09$ (5H, H4), 4.08 $(2 \mathrm{H}, \mathrm{H} 3), 4.15\left(\mathrm{~m}, 2 \mathrm{H},{ }^{3} J_{\mathrm{PH}}=9.6 \mathrm{~Hz},{ }^{3} J_{\mathrm{HH}}=6.4 \mathrm{~Hz}\right.$, $\left.\mathrm{OCH}_{2}\right), 4.20(2 \mathrm{H}, \mathrm{H} 2) .{ }^{13} \mathrm{C} \mathrm{NMR}\left(\mathrm{CDCl}_{3}\right.$, ppm, numberings of carbons are given in Scheme 1): $\delta 25.09\left[\mathrm{NCH}_{2} \mathrm{CH}_{2} \mathrm{CH}_{2}\right.$ (pip)], $25.22\left[\mathrm{NCH}_{2} \mathrm{CH}_{2} \mathrm{CH}_{2}\right.$ (pip)], $26.30\left[\mathrm{~d},{ }^{3} \mathrm{~J}_{\mathrm{PC}}=6.8 \mathrm{~Hz}\right.$, $\mathrm{NCH}_{2} \mathrm{CH}_{2}$ (pip)], $26.56\left[\mathrm{~d},{ }^{3} \mathrm{~J}_{\mathrm{PC}}=7.6 \mathrm{~Hz}, \mathrm{NCH}_{2} \mathrm{CH}_{2}\right.$ (pip)], 45.23 (C5), 45.17 [ $\mathrm{NCH}_{2}$ (pip)], 45.49 [ $\mathrm{NCH}_{2}$ (pip)], $46.16\left(\mathrm{~d},{ }^{2} J_{\mathrm{PC}}=13.7 \mathrm{~Hz}, \mathrm{NCH}_{2}\right), 63.23\left(\mathrm{OCH}_{2}\right), 68.34$ (C4), 68.05 (C3), 69.65 (C2), $84.36\left(\mathrm{~d},{ }^{3} J_{\mathrm{PC}}=11.5 \mathrm{~Hz}, \mathrm{C} 1\right)$.

2.5j Synthesis of 15: The procedure used for 11 was followed; using 5 (0.75 g, $1.37 \mathrm{mmol})$, piperidine $(1.08 \mathrm{~mL}$, $10.95 \mathrm{mmol})$ and triethylamine $(1.54 \mathrm{~mL})$. The first product eluted was the geminal-piperidino-substituted derivative (10). Yield: $0.19 \mathrm{~g}(22 \%)$. The second product was the tetrakispiperidino-substituted derivative (15). Yield: $0.71 \mathrm{~g}(70 \%)$, M.p. $218{ }^{\circ} \mathrm{C}$. Anal. Calc. for $\mathrm{C}_{34} \mathrm{H}_{57} \mathrm{~N}_{8} \mathrm{OFeP}_{3}$ : C, 54.99; H, 7.74; N, 15.09. Found: C, 55.26; H, 7.59; N, 14.81. APIESMS (fragments were based on $\left.{ }^{56} \mathrm{Fe}, \operatorname{Ir} \%\right): m / z 743\left([\mathrm{MH}]^{+}\right.$, 
100.0). FTIR (KBr, cm ${ }^{-1}$ ): 3089 (C-H arom.), 2954, 2844 (C-H aliph.), $1193(\mathrm{P}=\mathrm{N}) .{ }^{1} \mathrm{H} \mathrm{NMR}\left(\mathrm{CDCl}_{3}\right.$, ppm, numberings of protons are given in Scheme 1): $1.59[\mathrm{~m}, 8 \mathrm{H}$, $\mathrm{NCH}_{2} \mathrm{CH}_{2} \mathrm{CH}_{2}$ (pip)], 1.58 [m, 16H, $\mathrm{NCH}_{2} \mathrm{CH}_{2}$ (pip)], 1.73 $\left[\mathrm{m}, 2 \mathrm{H},{ }^{3} J_{\mathrm{HH}}=5.6 \mathrm{~Hz},{ }^{3} J_{\mathrm{HH}}=5.6 \mathrm{~Hz}, \mathrm{NCH}_{2} \mathrm{CH}_{2}\right.$ (spiro)], 2.89-3.04 (m, 2H, $\left.{ }^{3} J_{\mathrm{HH}}=5.6 \mathrm{~Hz}, \mathrm{NCH}_{2}\right), 2.89-3.04[\mathrm{~m}$, $16 \mathrm{H}, \mathrm{NCH}_{2}$ (pip)], 3.73 (d, 2H, $\left.{ }^{3} J_{\mathrm{PH}}=6.4 \mathrm{~Hz}, \mathrm{H} 5\right), 4.08$ $(5 \mathrm{H}, \mathrm{H} 4), 4.07(1 \mathrm{H}, \mathrm{H} 3), 4.17\left(\mathrm{~m}, 2 \mathrm{H},{ }^{3} J_{\mathrm{PH}}=10.8 \mathrm{~Hz}\right.$, $\left.{ }^{3} J_{\mathrm{HH}}=5.4 \mathrm{~Hz}, \mathrm{OCH}_{2}\right), 4.21(1 \mathrm{H}, \mathrm{H} 2) .{ }^{13} \mathrm{C} \mathrm{NMR}\left(\mathrm{CDCl}_{3}\right.$, ppm, numberings of carbons are given in Scheme 1): $\delta 25.16$ [ $\mathrm{NCH}_{2} \mathrm{CH}_{2} \mathrm{CH}_{2}$ (pip)], $25.24\left[\mathrm{NCH}_{2} \mathrm{CH}_{2} \mathrm{CH}_{2}\right.$ (pip)], 26.33 $\left[\mathrm{d},{ }^{3} \mathrm{~J}_{\mathrm{PC}}=7.7 \mathrm{~Hz}, \mathrm{NCH}_{2} \mathrm{CH}_{2}\right.$ (pip)], $26.55\left[\mathrm{~d},{ }^{3} J_{\mathrm{PC}}=\right.$ $7.1 \mathrm{~Hz}, \mathrm{NCH}_{2} \mathrm{CH}_{2}$ (pip)], $26.25\left(\mathrm{NCH}_{2} \mathrm{CH}_{2}\right), 45.23\left[\mathrm{NCH}_{2}\right.$ (pip)], 45.57 [ $\mathrm{NCH}_{2}$ (pip)], $45.39\left(\mathrm{NCH}_{2}\right), 47.78$ (C5), 65.69 $\left(\mathrm{d},{ }^{2} J_{\mathrm{PC}}=7.0 \mathrm{~Hz}, \mathrm{OCH}_{2}\right), 68.27(\mathrm{C} 4), 68.91$ (C3), 70.46 (C2), $83.96\left(\mathrm{~d},{ }^{3} J_{\mathrm{PC}}=14.1 \mathrm{~Hz}, \mathrm{C} 1\right)$.

\section{Results and Discussion}

\subsection{Syntheses}

The tetrachloro N/N (1-3) and N/O (4 and 5) spirocyclotriphosphazenes are the fertile starting compounds for the preparation of partly and fully-substituted phosphazenes. They have four reactive $\mathrm{Cl}$ atoms and they can give the substitution reactions with different mono, bidentate and multidentate ligands. Thus, the reactions of one equimolar amount of the compounds 1-5 with two equimolar amounts of piperidine afford the geminal-piperidino-substituted cyclotriphosphazenes (6-10), regioselectively. The thin-layer chromatography (TLC) exhibits no other compounds in the

\section{(a) Compound 9 Compound 14}
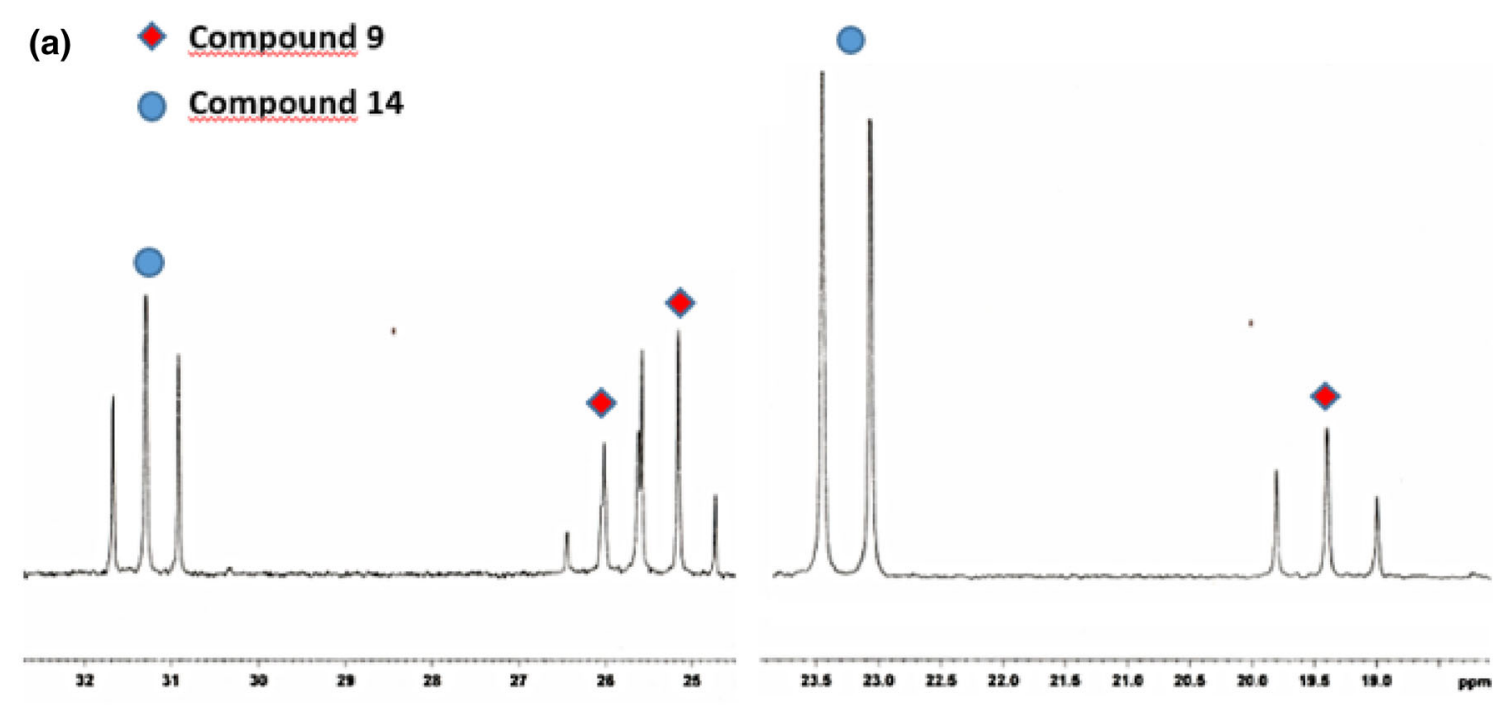

(b)

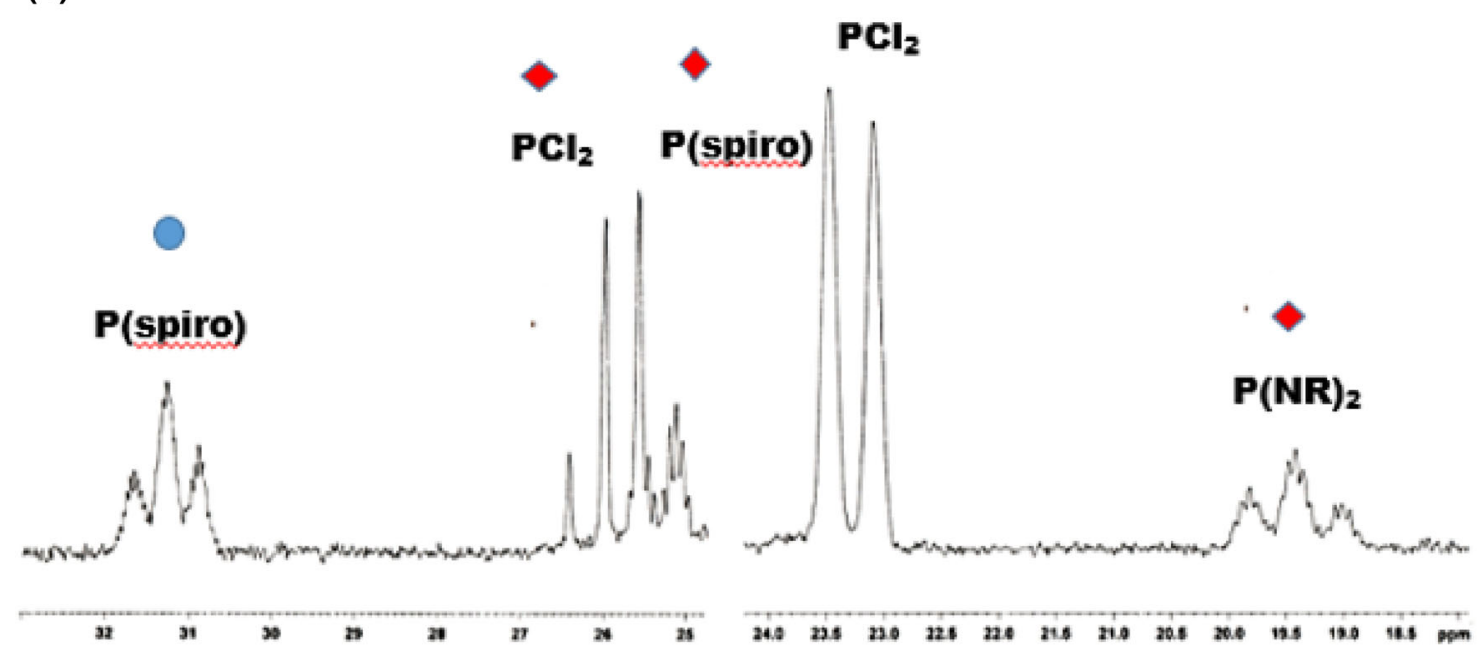

Figure 1. (a) ${ }^{1} \mathrm{H}$-decoupled and (b) coupled ${ }^{31} \mathrm{P}$ NMR spectra of 9 and14. 
Table 1. ${ }^{31} \mathrm{P}-\mathrm{NMR}$ (decoupled) spectral data of the phosphazenes. [Chemical shifts $(\delta)$ reported in $\left.\mathrm{Hz}\right]^{\mathrm{a}}$.
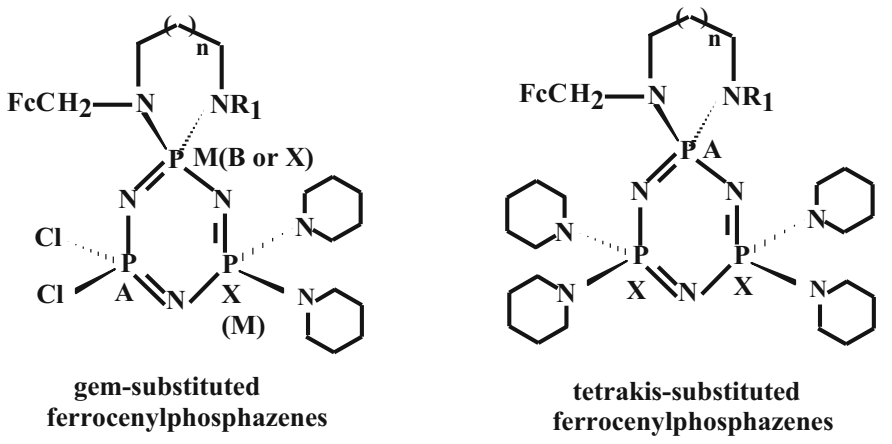

\begin{tabular}{llllll}
\hline \multirow{2}{*}{ Compound } & \multirow{2}{*}{ Spin System } & \multicolumn{3}{c}{$\delta(\mathrm{ppm})$} & \multirow{2}{*}{${ }^{2} \mathrm{~J}_{\mathrm{PP}}(\mathrm{Hz})$} \\
\cline { 3 - 5 } & & $\mathrm{PCl}_{2}$ & $\mathrm{P}(\mathrm{NR})_{2}(\mathrm{spiro})$ & $\mathrm{P}(\mathrm{NR})_{2}$ & \\
\hline 6 & AMX & $25.40(\mathrm{dd})$ & $22.06(\mathrm{dd})$ & $18.57(\mathrm{dd})$ & $41.3 ; 43.7 ; 51.0$ \\
7 & $\mathrm{AMX}$ & $25.26(\mathrm{dd})$ & $21.26(\mathrm{dd})$ & $18.60(\mathrm{dd})$ & $40.1 ; 43.7 ; 51.0$ \\
8 & $\mathrm{AMX}$ & $26.62(\mathrm{dd})$ & $18.26(\mathrm{dd})$ & $16.18(\mathrm{dd})$ & $43.7 ; 48.6 ; 51.9$ \\
9 & $\mathrm{ABX}$ & $26.03(\mathrm{dd})$ & $25.16(\mathrm{dd})$ & $19.41(\mathrm{dd})$ & $49.0 ; 50.4 ; 51.6$ \\
10 & $\mathrm{AMX}$ & $23.58(\mathrm{dd})$ & $13.90(\mathrm{dd})$ & $19.13(\mathrm{dd})$ & $39.2 ; 45.4 ; 52.9$ \\
11 & $\mathrm{AX}_{2}$ & - & $27.08(\mathrm{t})$ & $23.04(\mathrm{~d})$ & 40.1 \\
12 & $\mathrm{AX}_{2}$ & - & $27.02(\mathrm{t})$ & $23.04(\mathrm{~d})$ & 41.7 \\
13 & $\mathrm{AX}_{2}$ & - & $22.97(\mathrm{t})$ & $21.19(\mathrm{~d})$ & 36.4 \\
14 & $\mathrm{AX}_{2}$ & - & $31.62(\mathrm{t})$ & $23.26(\mathrm{~d})$ & 46.2 \\
15 & $\mathrm{AX}_{2}$ & - & $20.12(\mathrm{t})$ & $22.40(\mathrm{~d})$ & 43.8 \\
\hline
\end{tabular}

${ }^{\mathrm{a} 31} \mathrm{P}$ NMR measurements in $\mathrm{CDCl}_{3}$ solutions at $293 \mathrm{~K}$.

reaction mixture. The reaction yields of the compounds 6-10 are found to be in the range of $78-84 \%$. On the other hand, the reactions of one equimolar amount of the compounds 1-5 with excess piperidine give the geminal(6-10) and fully-piperidino-substituted cyclotriphosphazenes (11-15) (Scheme 1). The fully-substituted products (11-15) occur predominantly, and the reaction yields are calculated as $71,75,73,73$ and $70 \%$, respectively. In these reactions, triethylamine, $\mathrm{Et}_{3} \mathrm{~N}$, was used as an $\mathrm{HCl}$ acceptor. As an example, the coupled and decoupled ${ }^{31} \mathrm{P}$ NMR spectra of the reaction mixture of 4 with excess piperidine are depicted in Figure 1, showing that the geminal (9) and fully-piperidino-substituted ferrocenylphosphazenes (14) are present in the mixture.

Besides, each of the geminal products $(\mathbf{6}-\mathbf{1 0})$ contains one stereogenic P-centers and they ought to behave as the enantiomeric mixtures ( $\mathrm{R}$ and $\mathrm{S}$ ). Luckily, the structure of $\mathbf{6}$ is exactly solved by X-ray crystallography (see section 3.4), and its absolute configuration is found to be as $\mathrm{S}$.

The microanalysis, FTIR, APIES-MS and NMR data are coherent with the proposed structures of the compounds. The mass spectra of $6,7,8,9,11,13,14$ and 15 disclose the protonated molecular ion $\left([\mathrm{MH}]^{+}\right)$peaks, whereas the mass spectra of $\mathbf{1 0}$ and $\mathbf{1 2}$ exhibit the molecular ion peaks $\left(\mathrm{M}^{+}\right)$.

\subsection{NMR and IR spectroscopy}

The ${ }^{1} \mathrm{H}$-decoupled ${ }^{31} \mathrm{P}$ NMR data of the new phosphazenes $(\mathbf{6}-\mathbf{1 5})$ are given in Table 1 . The ${ }^{31} \mathrm{P}$ NMR spectra of 6, 7, 11, 12 and 14 are presented in Figure S1 (Supplementary Information). The spin systems of all the geminal (except 9) and tetrakis-piperidinosubstituted ferrocenylphosphazenes were designated as $\mathrm{AMX}$ and $\mathrm{AX}_{2}$, respectively. Besides, the compound 9 has an ABX spin system. The 12- line resonance pattern in the ${ }^{31} \mathrm{P}$ NMR spectrum of the geminal-substituted compounds (6-10) arise from the doublet of doublets for each phosphorus atom. As expected, the fullysubstituted phosphazenes (11-15) have a triplet for one $\mathrm{P}$ (spiro) atom and a doublet for two other $\mathrm{P}$ atoms. The ${ }^{1} \mathrm{H}$-coupled ${ }^{31} \mathrm{P}$ NMR spectra were also recorded in order to determine the geminal structures of the compounds 6-10. The $\delta \mathrm{P}($ spiro)-shifts of the geminaland tetrakis-piperidino-substituted phosphazenes, bearing the six-membered spiro-rings, are smaller than those of the five-membered ones. Moreover, the average ${ }^{2} J_{P P}$ coupling constant of $\mathbf{1 1 - 1 5}$ is $41.6 \mathrm{~Hz}$.

Taking into account the coupling constants, chemical shifts and multiplicities; the ${ }^{13} \mathrm{C}$ and ${ }^{1} \mathrm{H}$ NMR signals of the new phosphazenes are definitely assigned, and the ${ }^{13} \mathrm{C}$ and ${ }^{1} \mathrm{H}$ NMR spectra of the two phosphazenes 
Table 2. LB Film Parameters.

\begin{tabular}{lcccc}
\hline Compound & $\begin{array}{l}\text { Spreading Solution Volume } \\
\text { and Concentration }\end{array}$ & $\begin{array}{l}\text { Target Pressure } \\
(\mathrm{mN} / \mathrm{m})\end{array}$ & $\begin{array}{l}\text { Area per Molecule from } \\
\text { Isotherm }\left(\AA^{2} / \text { molecule }\right)\end{array}$ & $\begin{array}{c}\text { Deposition Type and } \\
\text { Deposition Cycles }\end{array}$ \\
\hline $\mathbf{3}$ & $62 \mu \mathrm{L} ; 3.5 \mathrm{mg} / \mathrm{mL}$ & 2.0 & 12.5 & $\mathrm{Z} ; 4$ \\
$\mathbf{1 2}$ & $62 \mu \mathrm{L} ; 3.6 \mathrm{mg} / \mathrm{mL}$ & 19.0 & 13.5 & $\mathrm{Z} ; 6$ \\
\hline
\end{tabular}

were given in Figures S2 and S3 (Supplementary Information). The two piperidino-groups bonded to the same phosphorus atom show two groups of $\mathrm{NCH}_{2} \mathrm{CH}_{2}$ $\underline{\mathrm{C}} \mathrm{H}_{2}, \mathrm{NCH}_{2} \underline{C H}_{2}$ and $\mathrm{NCH}_{2}$ (pip) signals with small separations in the ${ }^{13} \mathrm{C}$ NMR spectra. The methyl carbons, $\mathrm{NCH}_{3}$, of $\mathbf{6}, \mathbf{8}, \mathbf{1 1}$ and $\mathbf{1 3}$ were assigned between 31.43 and $36.46 \mathrm{~Hz}$. The ferrocenyl carbon peaks, C1, $\mathrm{C} 2, \mathrm{C} 3$ and $\mathrm{C} 4$, were also determined in the range of $68.00-70.60 \mathrm{ppm}$. Additionally, the average ${ }^{2} J_{\mathrm{PC}}$ couplings between the $\mathrm{NCH}_{2}$ spiro-carbon and the $\mathrm{P}$ atoms of the phosphazenes containing the five-membered spiro rings $(\mathbf{6}, 7,9, \mathbf{1 1}, 12$ and 14$)$ is $13.0 \mathrm{~Hz}$. The ${ }^{3} J_{\mathrm{PC}}$ values between the ipso-C $(\mathrm{C} 1)$ and $\mathrm{P}$ atoms are observed for all the phosphazene derivatives, and the average ${ }^{3} J_{\mathrm{PC}}$ values of the geminal and tetrakis-compounds are calculated as $10.9 \mathrm{~Hz}$ and $12.5 \mathrm{~Hz}$, respectively.

The ${ }^{1} \mathrm{H}$ NMR spectra of $\mathbf{6 - 1 0}$ are highly complex because of the diastereotopic protons. The chemical shifts of $\mathrm{NC} \underline{H}_{3}$ protons are assigned between $2.50 \mathrm{ppm}$ and $2.53 \mathrm{ppm}$, and the average ${ }^{3} J_{\mathrm{PH}}$ value of all the ferrocenylphosphazenes is calculated as $11.6 \mathrm{~Hz}$. The $\mathrm{H} 5$ protons of the fully-substituted- phosphazenes (11-15) are determined at $c a .3 .75 \mathrm{ppm}$ as doublets. The average ${ }^{3} J_{\mathrm{PH} 5}$ value of all the phosphazenes is $6.2 \mathrm{~Hz}$.

\subsection{The Interpretations of FTIR, GAIR and HATR Spectra of LB films of 3 and $\mathbf{1 2}$}

The determination of the LB film deposition parameters given in Table 2 is very crucial. The area per molecule on the subphase may reveal information on the conformation adopted by the molecules. The values of the area of $\mathbf{3}$ and 12 were determined, extrapolating the most steeply rising part of the $\pi-\mathrm{A}$ isotherms to the zero pressure.

The $\pi-\mathrm{A}$ isotherm of $\mathbf{1 2}$ is depicted in Figure 2, as an example. The best value of the deposition pressure of $\mathbf{1 2}$ is found to be $16 \mathrm{mN} / \mathrm{m}$. Figure 3 illustrates the reduced area of the monolayer during the deposition of the LB films of $\mathbf{1 2}$ in the ranges of one and four layers during the four monolayers transferring. It was understood from the surface pressure-time $(\pi-t)$ and area-time (At) curves of $\mathbf{1 2}$ (Figure 4) that the monolayers of the molecules were considerably stable.

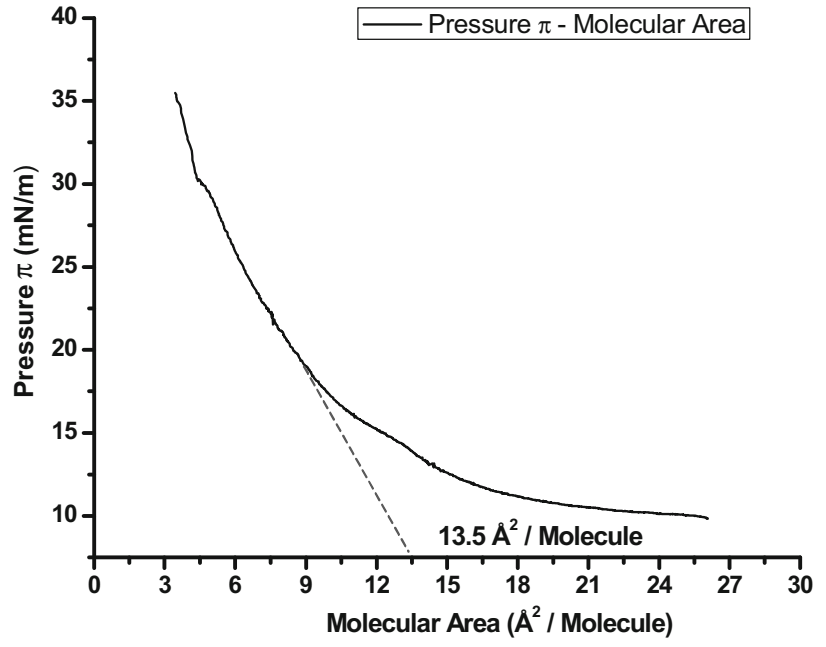

Figure 2. The surface pressure - area $(\pi-A)$ isoterm of 12.

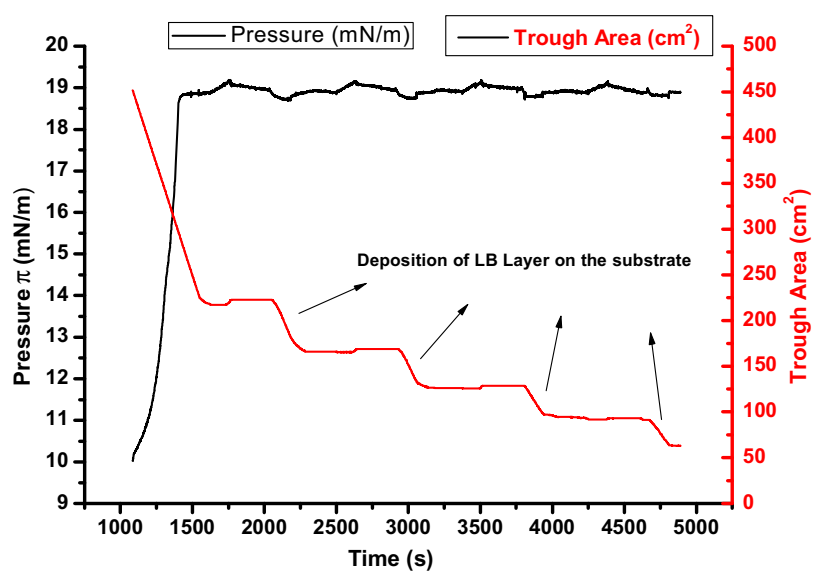

Figure 3. The reduced area of the monolayers during the deposition of the LB films of $\mathbf{1 2}$ between one and four layers, while four monolayers were transferring on to the substrate.

The transfer ratio (TR) is the best measure of transferring a Langmuir-Blodgett film to a substrate. This ratio is given by the following formula:

$\mathrm{TR}=$ (Area of monolayer removed from subphase at constant pressure)/(Area of substrate immersed in water)

The TR ought to be measured for each substrate pass through the air-water interface. Nevertheless, it must be kept in mind that the measured transfer rate is an 


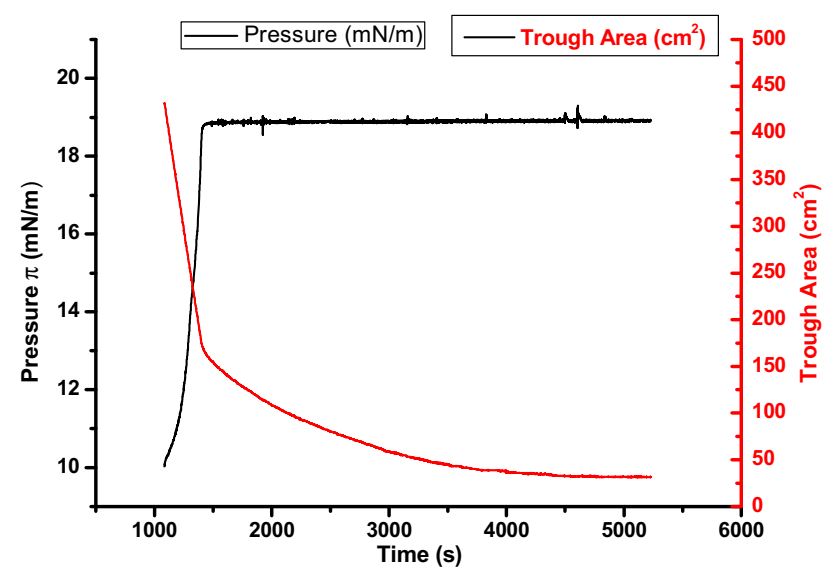

Figure 4. The $\pi-t$ and $A-t$ curves of monolayers of $\mathbf{1 2}$.

aggregate value over the entire immersed surface and ought to be interpreted most of the time. For instance, if there is $100 \%$ deposition on one side of the substrate and the film $100 \%$ is stripped from the other side, it is possible to measure a TR of $0 \%$.

The monolayers area of $\mathbf{1 2}$ removed from subphase at constant pressure (same for 4 layer) $\approx 40-42 \mathrm{~cm}^{2}$

The area of substrate [superforest (Cole-Parmer) 75 x $\left.\left.25 \times 1 \mathrm{~mm}^{3}\right)\right]$ immersed in water $=7.5 \mathrm{~cm} \mathrm{x} 2.5 \mathrm{~cm} \mathrm{x}$ 2 (two side immersed) $=37.5 \mathrm{~cm}^{2}$

For good deposition, TR should be 0.95-1.05. In compound 12, TR is calculated as 1.06 and greater than 1.00 because molecular stability changes when substrate immersed the subphase. This is nearly the same for all deposition cycle.

On the other hand, the deposition type $(\mathrm{Z})$ and deposition cycle values of $\mathbf{3}$ and $\mathbf{1 2}$ given in Table 2 are comparable to those of mono- and gem-DASDsubstituted phosphazene derivatives. ${ }^{25 b}$ The deposition type of four phosphazene derivatives is $\mathrm{Z}$, whereas the deposition cycles of the partly-substituted phosphazenes and the fully-substituted one (12) are found to be as 4 and 6 , respectively.

The FTIR spectra of the piperidinocyclotriphosphazenes were recorded on $\mathrm{KBr}$ pellet. The GAIR and HATR spectra of the LB ultrathin films of $\mathbf{3}$ and $\mathbf{1 2}$ were obtained. Figures 5, 6 and 7, respectively, illustrate the FTIR, p-polarized GAIR and HATR spectra of 12, as examples. In Table 3, the characteristic bands of 12 were given, and the characteristic p-polarized GAIR and HATR spectral data of $\mathbf{3}$ and $\mathbf{1 2}$ were also listed in Tables 4 and 5.

The asymmetric methyl $\left[\nu\left(\mathrm{CH}_{3}\right)\right]$ and methylene [ $\left.\nu\left(\mathrm{CH}_{2}\right)\right]$ stretching bands of $\mathbf{3}$ and $\mathbf{1 2}$ emerge at $c a$. 2963 and $2928 \mathrm{~cm}^{-1}$ in the FTIR spectra, respectively. In-plane bending frequencies of $\delta\left(\mathrm{CH}_{2}\right)$ and $\delta\left(\mathrm{CH}_{3}\right)$ are observed in the ranges of $1469-1329 \mathrm{~cm}^{-1}$ for $\mathbf{3}$

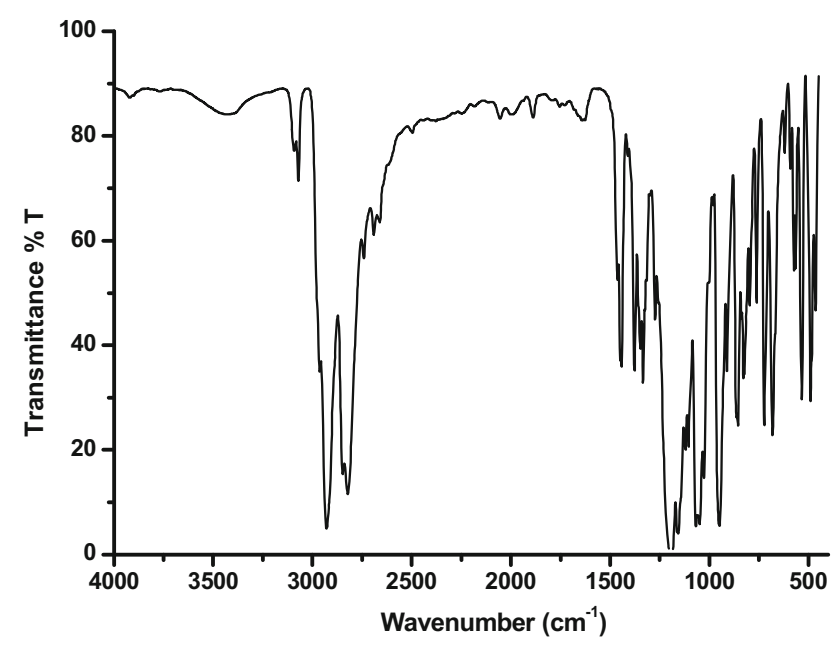

Figure 5. The infrared spectrum of $\mathbf{1 2}$ in a $\mathrm{KBr}$ pellet by transmission.

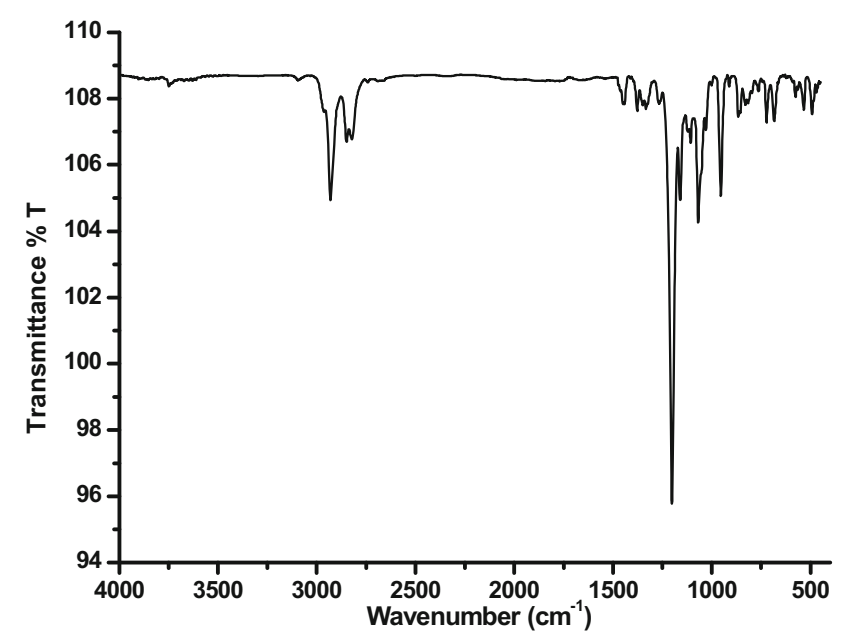

Figure 6. p-Polarized GAIR spectra of LB film of $\mathbf{1 2}$.

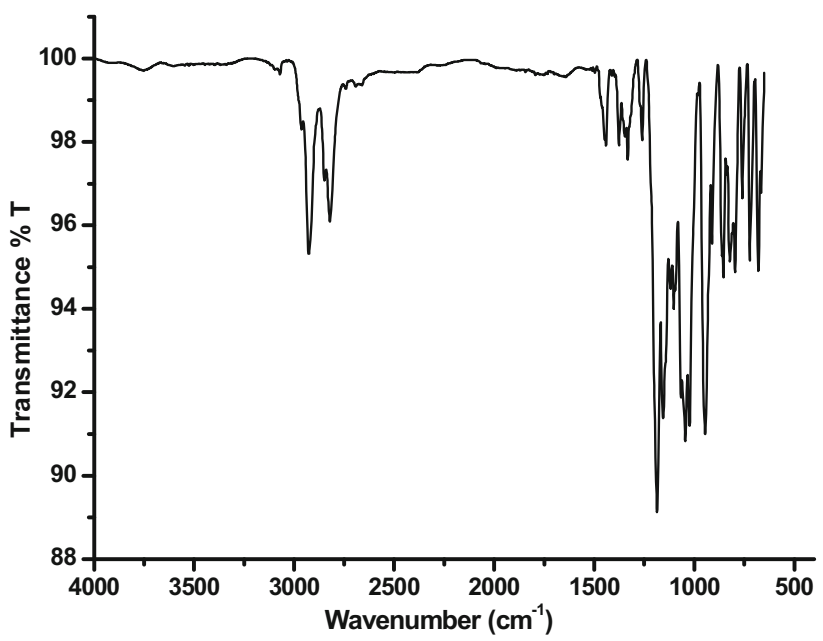

Figure 7. HATR spectra of LB film of $\mathbf{1 2 .}$

and $1448-1377 \mathrm{~cm}^{-1}$ for 12 . The characteristic $\nu(P=N)$ strong bands of the phosphazene rings are assigned at $1232 \mathrm{~cm}^{-1}, 1170 \mathrm{~cm}^{-1}$ and $860 \mathrm{~cm}^{-1}$ (for 3) and 
Table 3. The characteristic vibrational wavenumbers $\left(\mathrm{cm}^{-1}\right)$ of FTIR, GAIR and HATR spectra of 12.

\begin{tabular}{lccc}
\hline KBr Pellet (Solid Sample) $\left(\mathrm{cm}^{-1}\right)$ & GAIR of LB Films $\left(\mathrm{cm}^{-1}\right)$ & HATR of LB Films $\left(\mathrm{cm}^{-1}\right)$ & Assignments \\
\hline $2963 \mathrm{~s}$ & $2964 \mathrm{~m}$ & $2963 \mathrm{~m}$ & $\nu_{\mathrm{a}}\left(\mathrm{CH}_{3}\right)$ \\
$2928 \mathrm{~s}$ & $2930 \mathrm{~m}$ & $2927 \mathrm{~m}$ & $\nu_{\mathrm{a}}\left(\mathrm{CH}_{2}\right)$ \\
$2846 \mathrm{~s}$ & $2849 \mathrm{~m}$ & $2849 \mathrm{~m}$ & $\nu_{\mathrm{s}}\left(\mathrm{CH}_{2}\right)$ \\
$1448 \mathrm{~s}$ & $1450 \mathrm{w}$ & $1448 \mathrm{~m}$ & $\delta\left(\mathrm{CH}_{3}\right), \delta\left(\mathrm{CH}_{2}\right)$ \\
$1377 \mathrm{~s}$ & $1377 \mathrm{w}$ & $1377 \mathrm{~m}$ & $\delta\left(\mathrm{CH}_{3}\right), \delta\left(\mathrm{CH}_{2}\right)$ \\
$1200 \mathrm{~s}, \mathrm{~b}$ & $1201 \mathrm{~s}$ & $1187 \mathrm{~s}$ & $\nu(\mathrm{P}=\mathrm{N}),(\mathrm{CH})_{\text {ring }},(\mathrm{CC})_{\text {ring }}$ \\
$1156-1027 \mathrm{~s}$ & $1159-1028 \mathrm{~m}$ & $1154-1022 \mathrm{~s}$ & $(\mathrm{CH})_{\text {ring }},(\mathrm{CC})_{\text {ring }}$ \\
$861 \mathrm{~s}$ & $864 \mathrm{w}$ & $861 \mathrm{~m}$ & $\nu(\mathrm{P}=\mathrm{N})$ \\
\hline
\end{tabular}

$\nu$, stretching; $\delta$, in-plane bending; b, broad; w, weak; m, medium; s, strong; Subscript: a, asymmetric; s, symmetric.

Table 4. p-Polarized GAIR Bands for LB Films of $\mathbf{3}$ and $\mathbf{1 2 .}$

\begin{tabular}{|c|c|c|c|c|c|c|c|c|}
\hline \multirow{2}{*}{\multicolumn{2}{|c|}{$\begin{array}{l}\text { Compound IR Bands } \\
\qquad \begin{aligned} \text { Belongs } \\
\text { Rings }\left(\mathrm{cm}^{-1}\right) \\
\\
(\mathrm{CH}) \text { ve }(\mathrm{CC})\end{aligned}\end{array}$}} & \multicolumn{5}{|c|}{ Methyl and Methylene Groups $\left(\mathrm{cm}^{-1}\right)$} & \multicolumn{2}{|c|}{ IR Bands for Phosphazene Ring $\left(\mathrm{cm}^{-1}\right)$} \\
\hline & & $\overline{\nu_{a}\left(\mathrm{CH}_{3}\right)}$ & $v_{\mathrm{a}}\left(\mathrm{CH}_{2}\right)$ & $\nu_{\mathrm{s}}\left(\mathrm{CH}_{3}\right)$ & $v_{\mathrm{s}}\left(\mathrm{CH}_{2}\right)$ & $\delta\left(\mathrm{CH}_{2}\right)$ ve $\delta\left(\mathrm{CH}_{3}\right)$ & $\nu(\mathrm{P}=\mathrm{N})$ & $\nu_{\mathrm{a}}(\mathrm{P}-\mathrm{Cl})$ \\
\hline 3 & $1180-1003 \mathrm{~m}$ & $2963 \mathrm{~m}$ & $2923 w$ & - & $2855 \mathrm{w}$ & $1406,1351 \mathrm{w}$ & $1236,1180 \mathrm{~s}, 858 \mathrm{w}$ & - \\
\hline 12 & $1201-1028 \mathrm{~m}$ & $2964 \mathrm{~m}$ & $2930 \mathrm{~m}$ & - & $2849 m$ & $1450,1377 \mathrm{w}$ & $1201 \mathrm{~s}, 864 \mathrm{w}$ & - \\
\hline
\end{tabular}

$\nu$, streching; $\delta$, in-plane bending; b, broad; w, weak; m, medium; s, strong; Subscript: a, asymmetric; s, symmetric.

Table 5. HATR Bands for LB Films of $\mathbf{3}$ and $\mathbf{1 2 .}$

\begin{tabular}{|c|c|c|c|c|c|c|c|c|}
\hline \multirow{2}{*}{\multicolumn{2}{|c|}{$\begin{array}{l}\text { Compound IR Bands } \\
\text { Belongs } \\
\text { Rings }\left(\mathrm{cm}^{-1}\right) \\
(\mathrm{CH}) \text { ve }(\mathrm{CC})\end{array}$}} & \multicolumn{5}{|c|}{ Methyl and Methylene Groups $\left(\mathrm{cm}^{-1}\right)$} & \multicolumn{2}{|c|}{ IR Bands for Phosphazene Ring $\left(\mathrm{cm}^{-1}\right)$} \\
\hline & & $\nu_{\mathrm{a}}\left(\mathrm{CH}_{3}\right)$ & $v_{\mathrm{a}}\left(\mathrm{CH}_{2}\right)$ & $v_{\mathrm{s}}\left(\mathrm{CH}_{3}\right)$ & $v_{\mathrm{s}}\left(\mathrm{CH}_{2}\right)$ & $\delta\left(\mathrm{CH}_{2}\right)$ ve $\delta\left(\mathrm{CH}_{3}\right)$ & $\nu(P=N)$ & $\nu_{\mathrm{a}}(\mathrm{P}-\mathrm{Cl})$ \\
\hline 3 & $1179-1003 \mathrm{~s}$ & 2958w & 2921w & - & $2850 \mathrm{sh}$ & - & $1234,1179,857 \mathrm{~m}$ & $657 \mathrm{~m}$ \\
\hline 12 & $1187-1022 \mathrm{~s}$ & $2963 \mathrm{~m}$ & $2927 \mathrm{~m}$ & - & $2849 \mathrm{~m}$ & $1448,1377 \mathrm{~m}$ & $1187 \mathrm{~s}, 861 \mathrm{~m}$ & - \\
\hline
\end{tabular}

$\nu$, streching; $\delta$, in-plane bending; b, broad; w, weak; m, medium; s, strong; Subscript: a, asymmetric; s, symmetric.

$1200 \mathrm{~cm}^{-1}$ and $861 \mathrm{~cm}^{-1}$ (for 12). The (CC) ring and $(\mathrm{CH})_{\text {ring }}$ vibrations are found to be in the ranges of 1150 $1062 \mathrm{~cm}^{-1}$ and 1156-1027 for 3 and 12, respectively. The bands at $c a .660 \mathrm{~cm}^{-1}$ and $430 \mathrm{~cm}^{-1}$ in accordance with the published literature values, ${ }^{34,35}$ and they may be attributed to characteristic asymmetric and symmetric stretching frequencies of $\nu(\mathrm{P}-\mathrm{Cl})$, respectively.

The GAIR and HATR spectra are excellent data for investigating the orientation of ultrathin films at the molecular level. ${ }^{36}$ The comparative p-polarized GAIR and HATR spectral data of $\mathbf{3}$ and $\mathbf{1 2}$ were listed in Tables 4 and 5, indicating the clear 'coupling modes' of $\nu(\mathrm{P}=\mathrm{N})$ stretching bands at both spectra. As expected, the asymmetric $\nu(\mathrm{P}-\mathrm{Cl})$ stretching band of $\mathbf{3}$ is observed at $657 \mathrm{~cm}^{-1}$ in the HATR spectrum, implying that the $\mathrm{PCl}_{2}$ groups of LB films of 3 parallel oriented to the substrate surface. The $\nu(\mathrm{P}=\mathrm{N})$ bands disclose at 1236 ,
1234, 1180 and $1179 \mathrm{~cm}^{-1} ; 1201$ and $1187 \mathrm{~cm}^{-1}$ for 3 and 12, respectively. As seen in Tables 4 and 5, methyl $\left[v\left(\mathrm{CH}_{3}\right)\right]$ and methylene $\left[v\left(\mathrm{CH}_{2}\right)\right]$ modes are interpreted in the ranges of 2963-2850 $\mathrm{cm}^{-1}$ (for 3) and 2964-2849 $\mathrm{cm}^{-1}$ (for 12). Compared to GAIR and HATR intensities of $v\left(\mathrm{CH}_{3}\right)$ and $\nu\left(\mathrm{CH}_{2}\right)$ bands, both of the band modes are likely to be nearly parallel to the substrate surface for 12. But, in $\mathbf{3}$, these bands appear dominant on p-polarized GAIR spectrum, and they ought to be nearly parallel to the normal of the surface. The $\delta\left(\mathrm{CH}_{2}\right)$ and $\delta\left(\mathrm{CH}_{3}\right)$ modes were found to be at 1406 and $1351 \mathrm{~cm}^{-1}$ (for 3), and 1450 and $1377 \mathrm{~cm}^{-1}$ (for 12). Eventually, the spectral data of 3 and 12 indicate that both of the phosphazenes were deposited with the monolayers on the aluminium covered glass substrates as the $\mathrm{Z}$ deposited ultrathin $\mathrm{LB}$ films. 


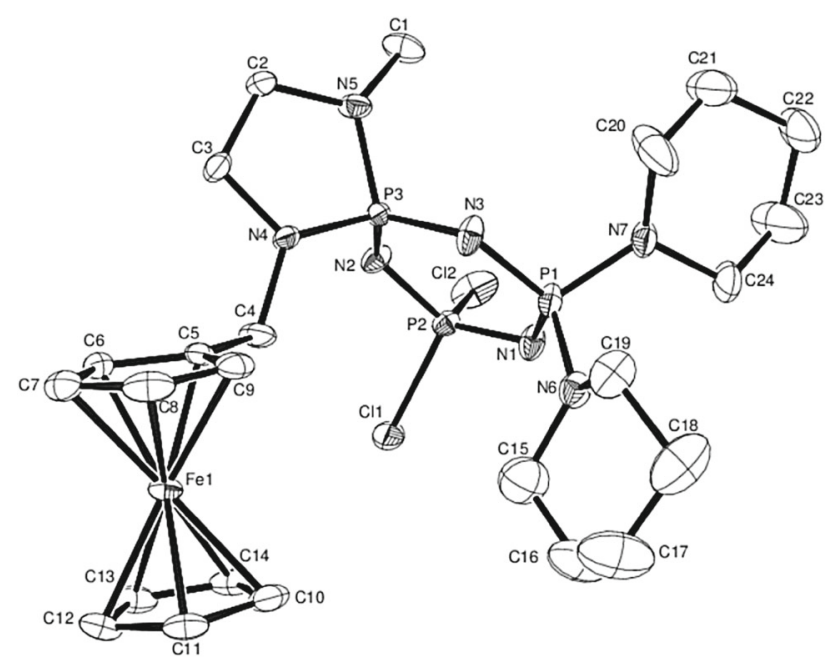

Figure 8. ORTEP- $3^{41}$ drawing of 6 with the atom numbering scheme. Displacement ellipsoids are drawn at the $30 \%$ probability level.

\section{$3.4 X$ X-Ray structure of the compound 6}

The molecular and crystal structure of 6 was established using X-ray crystallography. The ORTEP diagram of $\mathbf{6}$ is depicted in Figure 8 with the atom-numbering. The

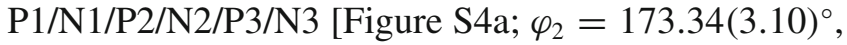
$\left.\theta_{2}=44.66(2)^{\circ}\right]$ phosphazene ring of $\mathbf{6}$ is nearly planar with total puckering amplitude $\mathrm{Q}_{\mathrm{T}}$ of $0.068(0.002)$ $\AA .{ }^{37}$ The five-membered spiro-ring (P3/N4/C3/C2/N5) is also almost planar (Figure $\mathrm{S} 4 \mathrm{~b}$, Supplementary Information). Compound 6 has one chiral P-center, and the chirality at $\mathrm{P} 3$ is $\mathrm{S}$. The space group is $\mathrm{C} 2 / \mathrm{c}$. It is among the Sohncke space groups, implying that only one enantiomer of $\mathbf{6}$ is crystallized from the racemic mixture. The chiral and non-chiral compounds crystallized in the well-known Sohncke space groups are discussed in the literature. ${ }^{38}$

The endocyclic N2-P3-N3 $\left[113.18(15)^{\circ}\right]$ and N1$\mathrm{P} 1-\mathrm{N} 3\left[115.46(16)^{\circ}\right]$ bond angles of 6 are narrow, whereas P1-N3-P3 [126.94(19) ${ }^{\circ}$ bond angle expands with respect to the corresponding values in the standard compound, $\mathrm{N}_{3} \mathrm{P}_{3} \mathrm{Cl}_{6} \cdot{ }^{39}$ On the other hand, there are regular variations with the distances from $\mathrm{P} 2: \mathrm{P} 2-\mathrm{N} 2 \approx$ $\mathrm{P} 2-\mathrm{N} 1<\mathrm{P} 3-\mathrm{N} 3 \approx \mathrm{P} 1-\mathrm{N} 3<\mathrm{P} 3-\mathrm{N} 2=\mathrm{P} 1-\mathrm{N} 1$. The average endocyclic $\mathrm{P}-\mathrm{N}$ bond length [1.588(3) $\mathrm{A}$ ] in the phosphazene ring is considerably shorter than the average exocyclic P-N bond length [1.636(3) Å]. Consequently, the bond lengths and bond angles values are in good agreement with the reported data in the literature. $^{2,40}$

\subsection{Antimicrobial activity}

In vitro antimicrobial activities of $\mathbf{6 , 7 , 9 , 1 0 , 1 1 , 1 2 , ~}$ 14 and 15 were studied against eight different bacteria and three fungi (Table S2, Supplementary Information). Ampicillin and chloramphenicol were used as standard antibacterial agents, Ketaconazole was used as an antifungal control. All the compounds have generally weak activities against the tested strain. However, the compound $\mathbf{1 1}$ has activity against all of the yeast strains. In addition, the compounds 9, 11 and 15 exhibit activity as Ketoconazole against C. albicans ATCC 10231 at high concentration. Consequently, according to the results given in Table S2 (Supplementary Information), most of these phosphazenes show weak to mild inhibition against tested bacterial and fungal strain.

The findings obtained in this paper are comparable with the literature data. It was found that the pyrrolidino and 1,4-dioxa-8-azaspiro[4,5]decane(DASD)-substituted cyclotriphosphazenes bearing ferrocenyl spirocyclic pendant arms were found to be active against some bacteria and fungi. ${ }^{3 a, 25 b, 33,42}$ While, tetra-morpholinoferrocenylphosphazenes exhibited no effect against these bacteria. ${ }^{27 b}$ Consequently, the antibacterial and antifungal activities seem to be considerably depending on the formation of hydrogen bonds with the substituents (pyrrolidino, morpholino, DASD and piperidino groups) bonded to the $\mathrm{P}$-atoms and the active centers of bacterial cell components.

\subsection{Interactions of DNA with the compounds 6, 7, 9, $10,12,14$ and 15}

The interactions of the phosphazenes $6,7,9,10,12,14$ and 15 with supercoiled DNA were investigated using the agarose gel electrophoresis. Figure 9 shows the electrophoretic mobility of the closed circular form I and open circular form II bands for the incubated mixtures of plasmid DNA at various concentrations of the compounds. In the electrophoretograms Lane $\mathrm{P}$ applies to untreated plasmid DNA as a control and Lanes 1-4 apply to plasmid DNA incubated with the compounds ranging from $2500 \mu \mathrm{M}$ to $312 \mu \mathrm{M}$. The presence of the smaller bands from form I and bigger bands from form II was observed in $\mathbf{9}, \mathbf{1 0}, \mathbf{1 2}, \mathbf{1 4}$ and 15 in the concentration range tested. The decreasing intensities of form I were also observed in compound $\mathbf{1 2}, \mathbf{1 4}$, and $\mathbf{1 5}$. Compounds $\mathbf{6}$ and $\mathbf{7}$ are found to be more damaging to plasmid DNA, causing DNA cleavage (existence of form III linear DNA in Lanes 1 and 2) and increased the mobilities and intensities of form II of the DNA. In addition, there is no form I DNA at the highest concentrations of $\mathbf{6}$ and 7. As shown in Figure 9, at a concentration above $625 \mu \mathrm{M}$, the compound $\mathbf{6}$ shows the DNA nuclease activity which converts supercoiled form I DNA into the linear form III DNA. The nuclease activity does not occur when DNA incubated with either $625 \mu \mathrm{M}$ or $312 \mu \mathrm{M}$ of $\mathbf{6}$. It 

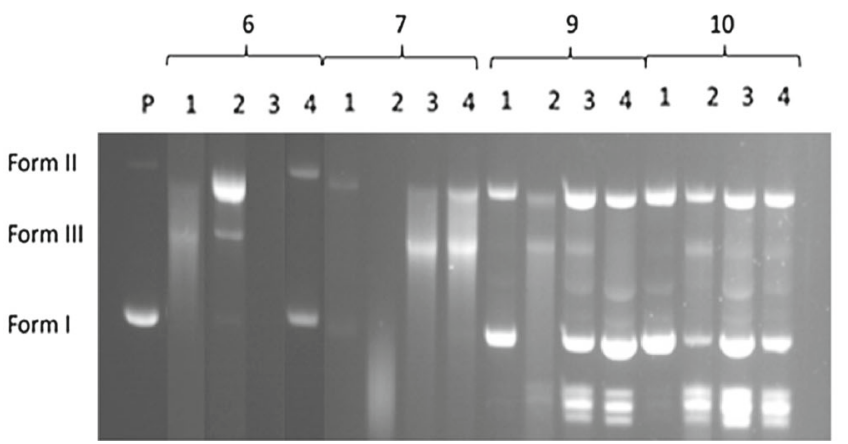

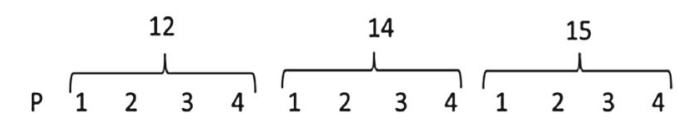

Form II

Form III

Form I

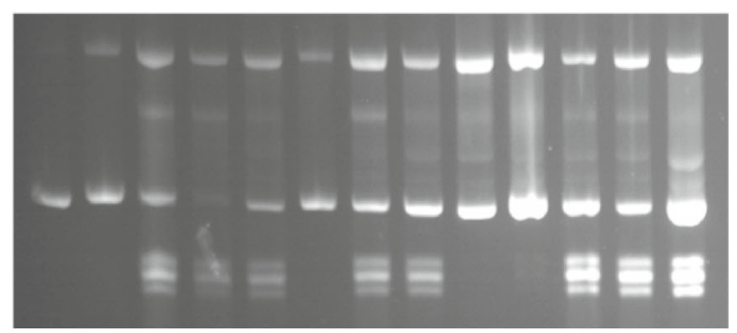

Figure 9. Gel electrophoretic mobility of plasmid DNA when incubated with various concentrations of 6, 7, 9, 10, 12, 14 and 15 (P: untreated plasmid DNA, Lanes 1-4: DNA treated by compounds at the concentration as follows: $2500,1250,625$ and $312 \mu \mathrm{M}$ ). The top and the bottom bands correspond to Form II (open circular) and Form I (covalently closed circular) and form III (linear), respectively.

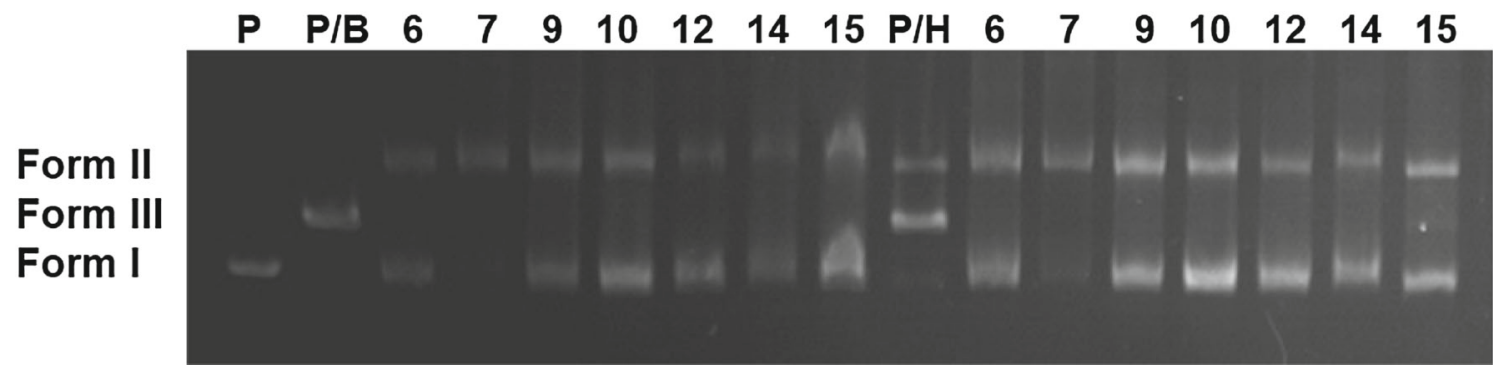

Figure 10. The electrophoretograms for BamHI (P/B) and HindIII (P/H) digested mixtures of DNA after treatment with the compounds. Lane P applies to untreated plasmid DNA and the other lanes apply to BamHI and HndIII digestion of untreated DNA.

is apparent that the compound $\mathbf{6}$ has begun to damage the DNA, causing it to lose its compact form and move slower through the gel and all of the plasmid DNA has been converted to the nicked form (Lane 4). Subsequent lanes begin to show a smear on the gel indicative of DNA double-strand cleavage. In the case of compound $\mathbf{7}$, as shown in Figure 9, at all concentration tested, compound shows DNA nuclease activity which converts supercoiled form I DNA into the linear form III DNA. It is apparent that the compound $\mathbf{7}$ has strong DNA damage, causing it to lose its compact form and slow mobility through the gel and all of the plasmid DNA has been converted to the nicked form (Lanes 3 and 4). Subsequent lanes also exhibit a smear on the gel indicative of DNA double-strand cleavage (Lanes 1 and 2). Similar results were found to be for the analogous DASD-substituted ferrocenylphosphazene derivatives. ${ }^{3 a}$ As known, the mobility and conformational changes may exhibit the binding activity of the compounds with hydrogen bonding and/or intercalation through the active sites of the DNA.

\subsection{Restriction digest analysis}

In order to assess the compounds $6,7,9,10,12,14$ and $\mathbf{1 5}$ showing affinity toward guanine-guanine (GG) and/or adenine-adenine (AA) regions, the restriction endonuclease analyses of the DNA-compound incubated mixtures by HindIII and BamHI enzymes for $1 \mathrm{~h}$ at the $37^{\circ} \mathrm{C}$ are carried out. When plasmid DNA was digested with BamHI or HindIII only one band corresponding to linear (form III) band was formed, whereas in the undigested plasmid DNA generally two bands corresponding to supercoiled and partially nicked form I and form II were observed. When plasmid DNA interacted with the compounds $6,7,9,10,12,14$ and 15 followed by $\mathrm{BamHI}$ digestion, two bands corresponding to forms I and II were observed at all the compounds.

BamHI and HindIII enzymes bind the specific 5'A/AGCTT-3', 5'-G/GATCC-3' sequences and cleave the adenine and guanine sites, respectively, as a result, Form I and Form II converts to a linear form of DNA (Form III) (Figure 10, PB). Eventually, the results reveal that the enzymes do not cleave the DNA for all the compounds indicating these phosphazenes binding to $\mathrm{G} / \mathrm{G}$ and $\mathrm{A} / \mathrm{A}$ nucleotides

\section{Conclusions}

In this paper, the partly (6-10) and fullysubstituted (11-15) ferrocenyl pendant-armed 
spirocyclotriphosphazenes were prepared by the reactions of the unsymmetrical N/N (1-3) and N/O (4 and 5) spirocyclotriphosphazenes with piperidine. It is an important result that the formation of geminal-products occurs only when the partlysubstituted phosphazene compounds containing bulky ferrocenyl-pendant-armed group are reacted with the two equimolar amounts of piperidine. Despite all the efforts made in this study, the theoretical expected non-geminal products (cis and/or trans) could not be obtained. In addition, the fully-substituted phosphazenes (11-15) obtained in this paper ought to be strong phosphazene-bases as well. In the future studies, the phosphazene-bases can give the protic molten salts (PMOS) with inorganic and organic acids, and they can be used as phosphazenium base catalysts and ionic liquids. The preparations and the characterizations using GAIR and HATR techniques of ultrathin and highly ordered Langmuir-Blodgett films of $\mathbf{3}$ and 12 are the most important part of this paper. As known, the phosphazene thin films are likely to be useful components in electronic and optical devices. In addition, DNA and the phosphazenes were analyzed by agarose gel electrophoresis whih illustrated the ability of phosphazenes to break double-stranded DNA.

\section{Supplementary Information (SI)}

Crystallographic data for the structure reported herein have been deposited with the Cambridge Crystallographic Data Centre as Supporting Information, CCDC No. 1813168 (for 6). Copies of the data can be obtained through application to CCDC, 12 Union Road, Cambridge CB2 1EZ, UK. (fax: +44 1223336033 or e-mail: deposit@ccdc.cam.ac.uk or at http:// www.ccdc.cam.ac.uk). Listings of the ${ }^{31} \mathrm{P},{ }^{13} \mathrm{C}$ and ${ }^{1} \mathrm{H}$ NMR spectra of the some phosphazenes (Figures S1, S2 and S3), the ring conformations and packing diagram of $\mathbf{6}$ (Figure S4), selected the bond lengths $(\AA)$ and the angles (deg) for6 (Table S1), the antimicrobial activities of $\mathbf{6 , 7 , 9 , 1 0 , 1 1 , 1 2 , 1 4}$ and15 (Table S2), antimicrobial activities (Section S1) and DNA-compound interactions (Section S2). Supplementary Information is available at www.ias.ac.in/chemsci.

\section{Acknowledgements}

Z.K. thanks the Turkish Academy of Sciences (TÜBA) for partial support of this work. M.C. and T.H. acknowledge the financial support of this work by Hacettepe University, Scientific research Unit (Grant No: 013D04602004).

\section{Compliance with ethical standards}

\section{Conflicts of interest}

There are no conflicts of interest to declare.

\section{References}

1. (a) Stewart F F 2015 Organophosphorus Chemistry Vol. 44 (Cambridge, UK: Royal Society of Chemistry) Ch. 9 p. 397; (b) Gleria M and De Jaeger R 2004 Applicative Aspect of Cyclophosphazenes (New York: Nova Science Publishers); (c) Allen C W 1991 Regioand stereochemical control in substitution reactions of cyclophosphazenes Chem. Rev. 91119

2. (a) Bilge Koçak S, Koçoğlu S, Okumuş A, Kılıç Z, Öztürk A, Hökelek T, Ö́ner Y and Açık L 2013 Syntheses, spectroscopic properties, crystal structures, biological activities, and DNA interactions of heterocyclic amine substituted spiro-ansa-spiro- and spirobino-spiro-phosphazenes Inorg. Chim. Acta 406 160; (b) Okumuş A, Bilge S, Kılıç Z, Öztürk A, Hökelek T and Yilmaz F 2010 Phosphorus-nitrogen compounds. Part 20: Fully substituted spiro-cyclotriphosphazenic lariat (PNP-pivot) ether derivatives Spectrochim. Acta $A 76$ 401; (c) Satish Kumar N and Kumara Swamy K C 2004 Synthesis and structures of unsymmetrical bis- and tris-cyclotriphosphazenes Polyhedron $\mathbf{2 3}$ 979; (d) Kumaraswamy S, Vijjulatha M, Muthiah C, Kumara Swamy K C and Engelhardt U 1999 Synthesis, reactivity and structures of spirocyclic products derived from octachlorocyclotetraphosphazene: comparison with spirocyclic cyclotriphosphazenes and linear phosphazenes J. Chem. Soc., Dalton Trans. 891; (e) Işıklan M, Sayın L, Sonkaya Ö, Hökelek T, Türk M and Oğuztüzün S 2016 Synthesis, structural characterization, and cytotoxic activity of new spirocyclic octachlorocyclotetraphosphazenes Phosphorus, Sulfur Silicon Relat. Elem. 1911216

3. (a) Asmafiliz N, Kılıç Z, Hökelek T, Açık L, Koç L Y, Süzen Y and Öner Y 2013 Phosphorus-nitrogen compounds: Part 26. Syntheses, spectroscopic and structural investigations, biological and cytotoxic activities, and DNA interactions of mono and bisferrocenylspirocyclotriphosphazenes Inorg. Chim. Acta 400 250; (b) Tümer Y, Koç L Y, Asmafiliz N, Kılıç Z, Hökelek T, Soltanzade H, Açık L, Yola M L and Solak A O 2015 Phosphorusnitrogen compounds: part 30. Syntheses and structural investigations, antimicrobial and cytotoxic activities and DNA interactions of vanillinato-substituted NN or NO spirocyclic monoferrocenyl cyclotriphosphazenes J. Biol. Inorg. Chem. 20 165; (c) Egemen G, Hayvalı M, Kılıç Z, Solak A O and Üstündă Z 2010 Phosphorus-nitrogen compounds Part 17: The synthesis, spectral and electrochemical investigations of porphyrino-phosphazenes J. Porphyrins Phthalocyanines 14 227; (d) Beşli S, Mutlu C, İbişoğlu H, Yüksel F and Allen C W 2015 Synthesis of a New Class of Fused Cyclotetraphosphazene Ring Systems Inorg. Chem. 54 334; (e) Kilıç Z, Contractor S R and Shaw R A 1987 Phosphorus-nitrogen compounds. Part 50. Further studies on the formation of bicyclic cyclotetraphosphazatetraene derivatives 2023

4. (a) Beşli S, Mutlu Balcı C, Uslu A and Allen C 2017 Newsynthetic approach to molecular rods using cyclophosphazene-basedoligospiranes Inorg. Chem. 56 9413; (b) Bilge S, Demiriz Ş, Okumuş A, Kılıç Z, Tercan B, Hökelek T and Büyükgüngör O 2006 
Phosphorus-nitrogen Compounds. Part 13. Syntheses, crystalstructures, spectroscopic, stereogenic, and anisochronic propertiesof novel spiro-ansa-spiro-, spiro-bino-spiro-, and spiro-cryptaphosphazene derivatives Inorg. Chem. 45 8755; (c) Muralidharan K and Elias A 2003 Preparation of the first examples ofansa-spiro substituted fluorophosphazenes and their structuralstudies: Analysis of C-H ‥ F-P weakinteractions in substituted fluorophosphazenes J. Inorg. Chem. 42 7535; (c) Asmafiliz N, İlter E E, Kılıç Z, Hökelek T and Şahin E 2008 Phosphorus-nitrogen compounds:Part 15. Synthesis, anisochronism and the relationship betweencrystallographic and spectral data of monotopic spiro-cryptaphosphazenes J. Chem. Sci. 120 363; (d) İbişoğlu H, Yenilmez Çiftçi G, KılıçA, Tanrıverdi E, Ün İ, Dal H and Hökelek T 2009 Formation of novel spiro, spiroansa and dispiroansa derivatives ofcyclotetraphosphazene from the reactions of polyfunctional amineswith octachlorocyclotetraphosphazatetraene $J$. Chem. Sci. 121125

5. Çosut B, Hacıvelioğlu F, Durmuş M, Kılıç A and Yeşilot S 2009 The synthesis, thermal and photophysical properties of phenoxycyclotriphosphazenyl-substituted cyclic and polymeric phosphazenes Polyhedron $\mathbf{2 8}$ 2510

6. (a) Allcock H R 2006 Recent developments in polyphosphazene materials science Curr. Opin. Solid State Mater. Sci. 10 231; (b) Mark J E, Allcock H R and West R 2005 Inorg. Polym. (USA: Oxford University Press)

7. (a) Steiner A, Zacchini S and Richards P I 2002 From neutral iminophosphoranes to multianionic phosphazenates. The coordination chemistry of imino-azaP(V) ligands Coord. Chem. Rev. 227 193; (b) Chandrasekhar V and Nagendran S 2001 Phosphazenes as scaffolds for the construction of multi-site coordination ligands Chem. Soc. Rev. 30193

8. (a) Bhuvan Kumar N N and Kumara Swamy K C 2008 Single diastereomers of unsymmetrical tris-spirocyclic cyclotriphosphazenes based on 1, 1'-bi-2-naphtholSynthesis and structures Chirality 20 781; (b) Şahin Ün Ş 2014 Cyclotriphosphazenes having stereogenic phosphorus atoms: synthesis, characterization and their stereogenic properties Polyhedron 70 148; (c) Asmafiliz N 2014 Syntheses of chiral phosphazenes with stereogenic centers: NMR behavior in the presence of a chiral solvating agent Heteroatom Chem. 25 83; (d) Porwolik-Czomperlik I, Brandt K, Clayton T A, Davies D B, Eaton R J and Shaw R A 2002 Diastereoisomeric singly bridged cyclophosphazene-macrocyclic compounds Inorg. Chem. 41 4944; (e) Elmas G, Okumuş A, Kılıç Z, Çam M, Açık L and Hökelek T 2018 Phosphorus-Nitrogen Compounds. Part 40. The syntheses of (4-fluorobenzyl) pendant armed cyclotetraphosphazene derivatives: spectroscopic, crystallographic and stereogenic properties, DNA interactions and antimicrobial activities Inorg. Chim. Acta 476 110; (f) Elmas G, Okumuş A, Koç L Y, Soltanzade H, Kılıç Z, Hökelek T, Dal H, Açık L, Üstündağ Z, Dündar D and Yavuz M 2014 Phosphorus-nitrogen compounds. Part 29. Syntheses, crystal structures, spectroscopic and stereogenic properties, electrochemical investigations, antituberculosis, antimicrobial and cytotoxic activities and DNA interactions of ansa-spiro-ansa cyclotetraphosphazenes Eur. J. Med. Chem. 87662

9. Uslu A and Yeşilot S 2015 Chiral configurations in cyclophosphazene chemistry Coord. Chem. Rev. 29128

10. Beşli S, Allen C W, Mutlu Balcı C, Kara G, Yeşilot S and Uslu A 2017 Stereochemical aspects of cyclotriphosphazenes: Prochiral and pseudo-asymmetric phosphorus atoms Polyhedron 13549

11. Allcock $\mathrm{H}$ R, Napierala M E, Cameron $\mathrm{C} G$ and O'Connor S J M 1996 Synthesis and characterization of 1onically conducting alkoxy ether/alkoxy mixedsubstituent poly(organophosphazenes) and their use as solid solvents for 1onic conduction Macromolecules 29 1951

12. (a) Çil E, Tanyıldızı M A, Özen F, Boybay M, Arslan M and Görgülü O 2012 Synthesis, characterization, and biological-pharmacological evaluation of new phosphazenes bearing dioxybiphenyl and schiff base groups Arch. Pharm. Chem. Life Sci. 345 476; (b) Tümer Y, Asmafiliz N, Zeyrek C T, Kılıç Z, Açık L, Çelik S P, Türk M, Çağdaş Tunalı B, Ünver H and Hökelek T 2018 Syntheses, spectroscopic and crystallographic characterizations of cis- and trans-dispirocyclic ferrocenylphosphazenes: molecular dockings, cytotoxic and antimicrobial activities New J. Chem. 421740

13. (a) Brandt K, Bartczak T J, Kruszynski R and PorwolikCzomperlik I 2001 AIDS-related lymphoma screen results and molecular structure determination of a new crown ether bearing aziridinylcyclophosphazene, potentially capable of ion-regulated DNA cleavage action Inorg. Chim. Acta 322 138; (b) Berberoğlu İ, Asmafiliz N, Kılıç Z, Hökelek T, Koç L Y, Açık L, Türk M, Soltanzade H and Dal H 2016 Phosphorus nitrogen compounds: Part 34. Syntheses, structural investigations, cytotoxic and biological activities of spiroansa-spiro and spiro-bino-spiro tetrameric phosphazene derivatives Inorg. Chim. Acta 446 75; (c) Elmas G, Okumuş A, Sevinç P, Kılıç Z, Açık L, Atalan M, Türk M, Deniz G and Hökelek T 2017 Phosphorusnitrogen compounds. Part 37. Syntheses and structural characterizations, biological activities of mono and bis(4-fluorobenzyl)spirocyclotetraphosphazenes New J. Chem. 41 5818; (d) Elmas G, Okumuş A, Cemaloğlu R, Kılıç Z, Çelik S P, Açık L, Çağdaş Tunalı B, Türk M, Aytuna Çerçi N, Güzel R and Hökelek T 2017 Phosphorus-nitrogen compounds. part 38. Syntheses, characterizations, cytotoxic, antituberculosis and antimicrobial activities and DNA interactions of spirocyclotetraphosphazenes with bis-ferrocenyl pendant arms J. Organomet. Chem. 853 93; (e) Koran K, Tekin Ç, Biryan F, Tekin S, Sandal S and Görgülü A O 2017 Synthesis, structural and thermal characterizations, dielectric properties and in vitro cytotoxic activities of new 2,2,4,4-tetra (4'-oxy-substituted-chalcone)-6, 6diphenylcyclotriphosphazene derivatives Med. Chem. Res. 26962

14. Zhu J, Liu W, Chu R and Meng X 2007 Tribological properties of linear phosphazene oligomers as lubricants Tribology Int. 4010

15. Singh A, Krogman N R, Sethurman S, Nair L S, Sturgeon J L, Brown P W, Laurencin C T and Allcock H R 2006 Effect of side group chemistry on 
the properties of biodegradable L-alanine cosubstituted polyphosphazenes Biomacromolecules 7914

16. Greish Y E, Bender J D, Lakshmi S, Brown P W, Allcock $\mathrm{H}$ R and Laurencin C T 2005 Low temperature formation of hydroxyapatite-poly(alkyl oxybenzoate)phosphazene composites for biomedical applications Biomaterials $\mathbf{2 6}$ 1

17. Klein R, Welna D T, Weikel A, Allcock H R and Runt J 2007 Counterion effects on 1on mobility and mobile 1on concentration of doped polyphosphazene and polyphosphazene ionomers Macromolecules 403990

18. Yeşilot S, Çoşut B, Ardıç Alidağı H, Hacıvelioğlu F, Özpınar G A and Kılıç A 2014 Intramolecular excimer formation in hexakis(pyrenyloxy)cyclotriphosphazene: photophysical properties, crystal structure, and theoretical investigation Dalton Trans. 433428

19. Cao R and Diaz-Garcia A 2009 Coordination compounds built on metal surfaces. Coord. Chem. Rev. 2531262

20. (a) Chandrasekhar V, Andavan G T S, Nagendran S, Krishnan V, Azhakar R and Butcher R J 2003 Cyclophosphazene hydrazides as scaffolds for multi-ferrocenyl assemblies: synthesis, structure, and electrochemistry Organometallics 22 976; (b) Şenkuytu E and Tanrıverdi Eçik E 2017 Octa-BODIPY derivative dendrimeric cyclotetraphosphazenes; photophysical properties and fluorescent chemosensor for $\mathrm{Co}^{2+}$ ions Spectrochim. Acta Part A 173863

21. Barlow S and Marder S R 2000 Electronic and optical properties of conjugated group 8 metallocene derivatives Chem. Commun. 155

22. Barlow S, Bunting H E, Ringham C, Green J C, Bublitz G U, Boxer S G, Perry J W and Marder S R 1999 Studies of the electronic structure of metallocene-based secondorder nonlinear optical dyes J. Am. Chem. Soc. 1213715

23. (a) Gill R, Batool S, Qureshi R and Khalid S S 2015 Nanofabrication of Block Copolymers of PDMS/Polyamide having Trichlorogermyl Pendant Using LbL Technique J. Chem. Soc. Pak. 37 468; (b) Petty M C 1992 Possible applications for LangmuirBlodgett films Thin Solid Films 210-211 417

24. (a) Swalen J D, Allara D L, Andrade J D, Chandross E A, Garoff S, Israelachvili J, McCarthy T J, Murray R, Pease R F, Rabolt J F, Wynne K J and Yu H 1987 Molecular monolayers and films. A panel report for the Materials Sciences Division of the Department of Energy Langmuir 3 932; (b) Roberts G 1990 Langmuir-Blodgett Films (New York: Plenum Press)

25. (a) Asmafiliz N, Kılıç Z, Civan M, Avcı O, Gönder L Y, Açık L, Aydın B, Türk M and Hökelek T 2016 Phosphorus-nitrogen compounds. Part 36. Syntheses, Langmuir-Blodgett thin films and biological activities of spiro-bino-spiro trimeric phosphazenes New J. Chem. 40 9609; (b) Asmafiliz N, Civan M, Özben A, Kılıç Z, Ramazanoğlu N, Açık L and Hökelek T 2018 Appl. Organomet. Chem. 32 e4223

26. (a) Muralidharan K, Reddy N D and Elias A J 2000 Syntheses of Novel Exo and Endo Isomers of Ansa-Substituted Fluorophosphazenes and Their Facile Transformations into Spiro Isomers in the Presence of Fluoride Ions Inorg. Chem. 39 3988; (b) Myer C M and Allen C W 2002 N-(Ferrocenylmethyl)-Nmethylaminocyclotriphosphazenes Inorg. Chem. 41 60; (c) Sengupta S 2003 A hexaferrocenyl cluster based on a cyclotriphosphazene core: synthesis and electrochemistry Polyhedron 22 1237; (d) Sengupta S 2003 A ferrocene dendrimer based on a cyclotriphosphazene core Tetrahedron Lett. $\mathbf{4 4} 7281$

27. (a) Okumuş A, Akbaş H, Kılıç Z, Koç L Y, Açık L, Aydın B, Türk M, Hökelek $T$ and Dal H 2016 Phosphorus- nitrogen compounds: part 33: in vitro cytotoxic and antimicrobial activities, DNA interactions, syntheses and structural investigations of new mono(nitrobenzyl)cyclotriphosphazenes Res. Chem. Intermed. 42 4221; (b) Asmafiliz N, K1lıç Z, Öztürk A, Süzen Y, Hökelek T, Açık L, Çelik Z B, Koç L Y, Yola M L and Üstündağ Z 2013 Phosphorus-Nitrogen Compounds: Part 25. Syntheses, spectroscopic, structural and electrochemical investigations, antimicrobial activities, and DNA interactions of ferrocenyldiaminocyclotriphosphazenes Phosphorus Sulfur 188 1723; (c) Okumuş A, Elmas G, Kılıç Z, Ramazanoğlu N, Açık L, Türk M and Akça G 2017 The reactions of $\mathrm{N}_{3} \mathrm{P}_{3} \mathrm{Cl}_{6}$ with monodentate and bidentate ligands: the syntheses and structural characterizations, in vitro antimicrobial activities, and DNA interactions of 4-fluorobenzyl(N/O)spirocyclotriphosphazenes Turk. J. Chem. 41 525; (d) Elmas G, Okumuş A, Kılıç Z, Çelik S $P$ and Açık L 2017 The Spectroscopic and Thermal Properties, Antimicrobial Activities and DNA Interactions of 4-(Fluorobenzyl)Spiro(N/O) Cyclotriphosphazenium Salts J. Turk. Chem. Soc. Sect. A: Chem. 4993

28. Bruker program 1D WIN-NMR (release 6.0) and 2D WIN-NMR (release 6.1).

29. (a) Blaudez D, Buffeteau T, Desbat B, Orrit M and Turlet J M 1992 Characterization of Langmuir-Blodgett monolayers using polarization modulated FTIR spectroscopy Thin Solid Films 210-211 648; (b) Civan M, Topaçlı C, Topaçlı A, Gürol I and Ahsen V 2007 An infrared study on Langmuir-Blodgett films of 12,13-bis(hydroxyimino)-11,14-diazatetracosane $J$. Mol. Struct. 834-836 482

30. Bruker 2005 SADABS Bruker AXS Inc. Madison Wisconsin USA

31. Sheldrick G M 1997 SHELXS-97 SHELXL-97 University of Göttingen: Göttingen Germany

32. Sheldrick G M 2008 A short history of SHELX Acta Crystallogr. Sect. A 64112

33. İler E E, Asmafiliz N, Kılıç Z, Açık L, Yavuz M, Bali E B, Solak A O, Büyükkaya F, Dal H and Hökelek T 2010 Phosphorus-nitrogen compounds: Part 19. Syntheses, structural and electrochemical investigations, biological activities, and DNA interactions of new spirocyclic monoferrocenylcyclotriphosphazenes Polyhedron 292933

34. Carriedo G A, Alonso F G, Gonzalez P A and Menendez J R 1998 Infrared and Raman spectra of the phosphazene high polymer $\left[\mathrm{NP}\left(\mathrm{O}_{2} \mathrm{C}_{12} \mathrm{H}_{8}\right)\right]_{n}$ J. Raman Spectrosc. 29 327

35. Workman Jr J 2000 Handbook of Organic Compunds Methods and Interpretation (Location: Academic Press) p. 1

36. (a) Roeges N P G 1994 A Guide To The Complete Interpretation of Infrared Spectra of Organic Structure (Chichester UK: John Wiley); (b) Bellamy L J 1975 
The Infrared Spectra of Complex Molecules (New York: Chapman and Hall); (c) Mielczarski J A and Mielczarski E 1995 Determination of Molecular Orientation and Thickness of Self-Assembled Monolayers of Oleate on Apatite by FTIR Reflection Spectroscopy J. Phys. Chem. 993206

37. Cremer D and Pople J A 1975 General definition of ring puckering coordinates J. Am. Chem. Soc. 971354

38. Pidcock E 2005 Achiral molecules in noncentrosymmetric space groups Chem. Commun. 27 3457

39. Bullen G J 1971 An improved determination of the crystal structure of hexachlorocyclotriphosphazene (phosphonitrilic chloride) J. Chem. Soc. A 1450

40. Tümer Y, Asmafiliz N, Kılıç Z, Hökelek T, Koç L Y, Açık L, Yola M L, Solak A O, Öner Y, Dündar D and
Yavuz M 2013 Phosphorus-nitrogen compounds: Part 28. Syntheses, structural characterizations, antimicrobial and cytotoxic activities, and DNA interactions of new phosphazenes bearing vanillinato and pendant ferrocenyl groups J. Mol. Struct. 1049112

41. Farrugia L J 1997 ORTEP-3 for Windows - a version of ORTEP-III with a Graphical User Interface (GUI) J. Appl. Crystallogr. 30565

42. Asmafiliz N, Kılıç Z, Öztürk A, Hökelek T, Koç L Y, Açık L, Kısa Ö, Albay A, Üstündağ Z and Solak A O 2009 Phosphorus-nitrogen compounds. 18. Syntheses, stereogenic properties, structural and electrochemical investigations, biological activities, and DNA interactions of new spirocyclic mono- and bisferrocenylphosphazene derivatives Inorg. Chem. 48 10102 